Supplement of Clim. Past, 15, 1885-1911, 2019

https://doi.org/10.5194/cp-15-1885-2019-supplement

(C) Author(s) 2019. This work is distributed under

the Creative Commons Attribution 4.0 License.

(c) (1)

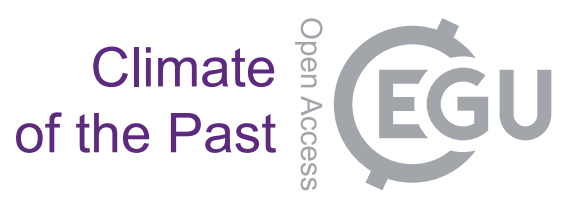

Supplement of

\title{
Effects of land use and anthropogenic aerosol emissions in the Roman Empire
}

Anina Gilgen et al.

Correspondence to: Anina Gilgen (anina.gilgen@env.ethz.ch) and Ulrike Lohmann (ulrike.lohmann@env.ethz.ch)

The copyright of individual parts of the supplement might differ from the CC BY 4.0 License. 


\section{S1 Map of the Roman Empire}

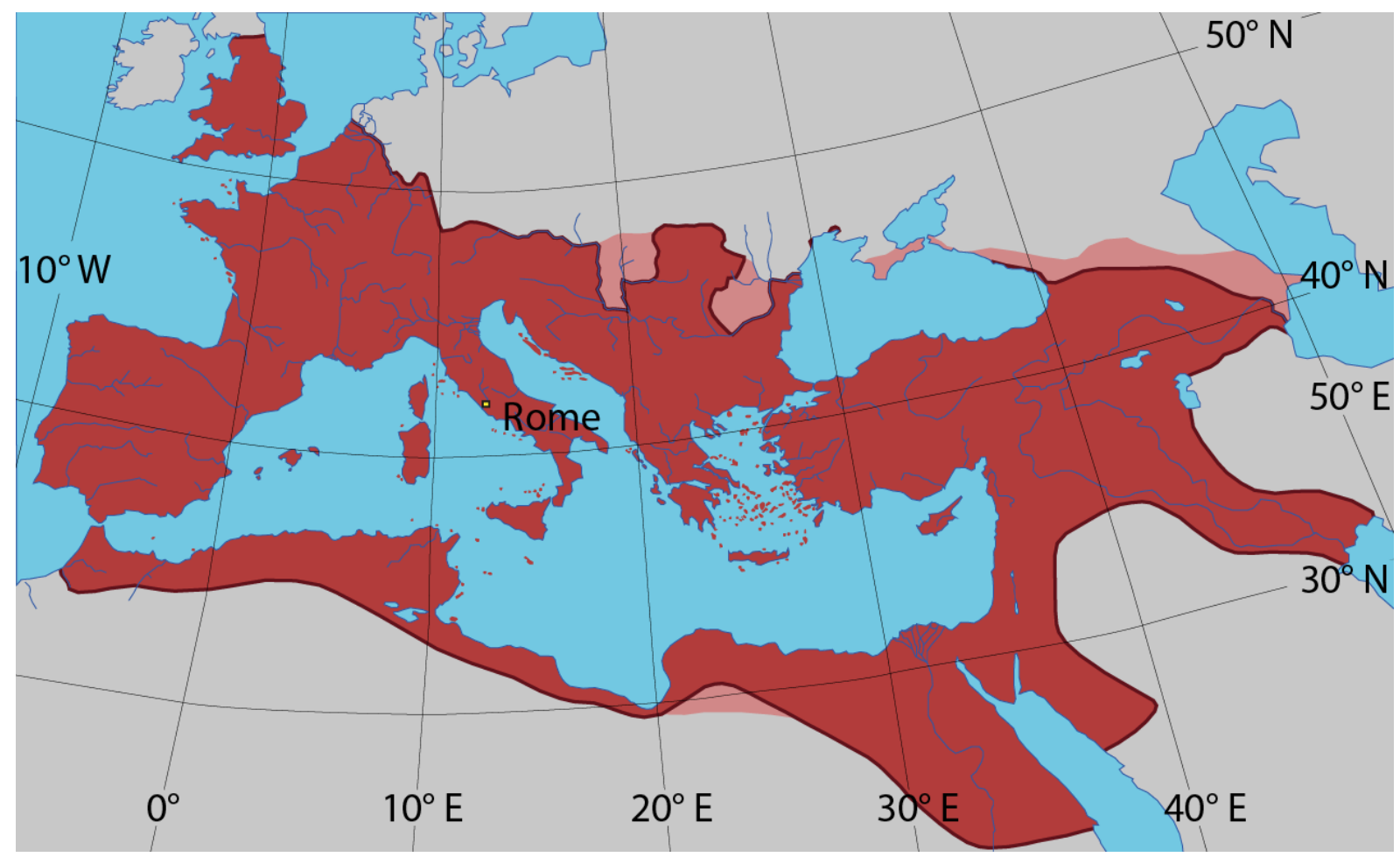

Fig. S1: The Roman Empire at its greatest extent $(117 \mathrm{CE})$ with its vassals in pink. Adapted from Tataryn (2016); licensed under the Creative Commons Attribution-Share Alike 3.0 Unported license (https://creativecommons.org/licenses/by-sa/3.0/deed.en). 


\section{S2 Model setup}

Tab. S1: Greenhouse gas concentrations and orbital parameters. Precession is expressed as the longitude of the perihelion with respect to the equinox. The values are averages over $50 \mathrm{CE}$ to $150 \mathrm{CE}$.

\begin{tabular}{l|l|c}
\hline Var. & Unit & Value \\
\hline $\mathrm{CO}_{2}$ & ppm & 278 \\
$\mathrm{CH}_{4}$ & ppb & 662 \\
$\mathrm{~N}_{2} \mathrm{O}$ & ppb & 267 \\
Eccentricity & - & 0.01742 \\
Precession & degrees & 250.8 \\
Obliquity & degrees & 23.68 \\
\hline
\end{tabular}

Tab. S2: Overview of natural fire aerosol, anthropogenic aerosol, and SOA precursor emissions in the different ECHAM-HAM-SALSA simulations. The multiplication factor refers to the natural fire emissions calculated with CBALONE-SPITFIRE. $l$ stands for the fraction of the vegetated area that is covered by anthropogenic land (crop and pasture) and AA for anthropogenic aerosol emissions.

\begin{tabular}{l|l|l|l}
\hline Simulation & $\begin{array}{l}\text { Multiplication } \\
\text { factor }\end{array}$ & AA & SOA precursor emissions \\
\hline no_human & 1 & No & based only on natural land cover \\
LCC_HYDE & 1 & No & changed due to anthropogenic land cover \\
LCC_KK & 1 & No & changed due to anthropogenic land cover \\
LCC_HYDE_low & $(1-l)$ & Yes & changed due to anthropogenic land cover \\
LCC_HYDE_int & $(1-l)$ & Yes & changed due to anthropogenic land cover \\
LCC_KK_high & $(1-l)$ & Yes & changed due to anthropogenic land cover \\
\hline
\end{tabular}




\section{S3 Aerosol emission factors for fuel consumption}

The fuel consumption studies that we considered mostly provide emission factors for either open fire places or traditional stoves. An exception is the measurement of olive pits that we included, which was conducted in a pellet stove; it was the only measurement of olive pits that we could find, and olive oil pressing waste was employed as a domestic and industrial fuel throughout Antiquity in the Mediterranean (Rowan, 2015).

\section{S3.1 Wood}

For wood combustion, we considered many wood types which were abundant in the Roman Empire (e.g. different types of oak, olive tree, maritime pine, or beech). However, many of these measurements were conducted with the same burning devices (Alves et al., 2011; Calvo et al., 2015 Fernandes et al., 2011). Looking at different studies, we observed that the differences in $E F$ s between different wood types are sometimes smaller than the differences between different measurement setups. Hence, we also decided to include some trees in our compilation which were not present in the Mediterranean region (e.g. Lespedeza, Paulownia) to consider more independent studies. The medians and quartiles that were derived for the emission factors of wood $\left(E F_{\text {wood }}\right)$ from the random sampling can be found in Table S3.

\section{S3.2 Agricultural waste}

For agricultural waste, a large variety of burning material was considered (e.g. wheat straw, rice straw, dung). Although the composition of agricultural residues in the Roman Empire differs from our compilation (e.g. rather Triticum dicoccum and barley instead of Triticum aestivum and rice; olive pits and chickpeas instead of peanuts and soybean residues), we assume that the emission factors are not fundamentally different: our compilation considers a large range of agricultural waste types. Furthermore, based on our compiled data, the measurement setup seems to be as important as the specific fuel type. The calculated medians and quartiles for the emission factors of agricultural waste $\left(E F_{\text {agri }}\right)$ can be found in Table $\mathrm{S} 4$.

\section{S3.3 Charcoal}

Compared to wood and agricultural waste, only few studies estimated aerosol $E F$ s for charcoal burning and charcoal making. Furthermore, most of them provide measurements of total suspended particles (e.g. Smith, 2000), and not for BC and OC. Thus, we considered only the very recent study by Keita et al. (2018) for BC and OC, who measured BC and OC for charcoal cooking fires and charcoal making in West Africa and calculated emission factors. Maybe even more important than knowing the exact $\mathrm{BC}$ and $\mathrm{OC}$ emission factors for charcoal burning is considering also the emissions from charcoal making. To produce $1 \mathrm{~kg}$ of charcoal, approximately $7 \mathrm{~kg}$ of wood are needed with traditional methods (Olson, 1991). As a consequence, using charcoal instead of wood as fuel might overall result in similar or even higher aerosol emissions, since for every kilogramme of charcoal burnt, the aerosols emitted during the production of this kilogramme of charcoal should also be considered. The aerosol emission factors per kilogramme of wood used for charcoal $\left(E F_{\mathrm{ch}_{\mathrm{w}}}\right)$ were therefore calculated as:

$$
E F_{\mathrm{ch}_{\mathrm{w}}}=\frac{1}{7} \cdot E F_{\mathrm{chb}}+E F_{\mathrm{chm}},
$$


where $E F_{\text {chb }}$ is the emission factor for charcoal burning (per kilogramme of charcoal) and $E F_{\text {chm }}$ is the emission factor for charcoal making (per kilogramme of wood). The emission factors for $E F_{\text {chb }}$ and $E F_{\text {chm }}$ can be found in Tables $\mathrm{S} 5$ and $\mathrm{S} 6$, respectively.

Considering that charcoal is often cited as almost smokeless (e.g. Wood and Baldwin, 1985 Lohri et al., 2016), the measured $E F_{\text {chb }}$ of BC (median: $0.59 \mathrm{~g} \mathrm{~kg}^{-1}$; comparable to the burning of other types of biofuel) is relatively high - a discrepancy that already Bond et al. (2004) noted for the emission factors of total suspended particles. In the future, more measurements could help to better understand this inconsistency.

Inserting the estimates of $E F_{\text {chb }}$ and $E F_{\text {chm }}$ in the equation above results in $E F_{\mathrm{ch}_{\mathrm{w}}}$ of $0.26 \mathrm{~g} \mathrm{~kg}_{\text {wood }}^{-1}(0.18,0.39)$ for BC, $3.94 \mathrm{~g} \mathrm{~kg}_{\text {wood }}^{-1}(3.27,4.80)$ for $\mathrm{OC}$, and $0.21 \mathrm{~g} \mathrm{~kg}_{\text {wood }}^{-1}(0.14$, 0.32 ) for $\mathrm{SO}_{2}$.

\section{S3.4 Combining different sectors}

To assess the overall emission factor, we needed to estimate how much which sector contributed to the total fuel consumption. Except for $\mathrm{SO}_{2}$, the emission factors are similar for wood and agricultural waste. The OC emission factors for charcoal burning and production (expressed as per kilogramme wood) are larger than for wood and agricultural waste, whereas the opposite is the case for BC. Thus, different assumptions concerning the contributions from the three sectors would affect the $\mathrm{BC}$ to $\mathrm{OC}$ ratio, rather than the emissions of both of them.

Wood fuel (including wood for charcoal making) was the dominant fuel in the Roman Empire (Olson, 1991). However, agricultural residues such as chaff, olive pits, and dung were also used, most evidently in regions lacking in supplies of wood (e.g. Roman North Africa and Roman East; Mietz, 2016 Rowan, 2015). In developing countries in 1985, the mass contribution of agricultural waste to total biofuel combustion (excluding burning in fields) ranges from $14 \%$ in Africa to over $40 \%$ in Asia (Yevich and Logan, 2003). Based on these numbers, we assumed that $20 \%$ of the used fuel consisted of agricultural waste.

Like for developing countries (Wood and Baldwin, 1985 Yevich and Logan, 2003 Lohri et al., 2016), the use of charcoal was especially important in urban areas in ancient times (Veal, 2017). Veal (2017) assumes that in the cities "perhaps $80 \%$ " of the burnt fuel consisted of charcoal with the remainder being wood, whereas the opposite ratio occurred in rural areas. In her two extreme case scenarios for Rome, she used charcoal contributions of $80 \%$ and $20 \%$ to total fuel. Assuming a conversion factor of 7 , this means that $97 \%$ and $64 \%$ of the wood fuel was used for charcoal making, respectively.

These estimates are higher than present-day estimates in countries where charcoal is produced: using data from the food and agricultural organisation $\left(\mathrm{FAO}^{1}\right)$, we calculated the contribution of charcoal production to total wood fuel production. We chose the year 1970, when fossil fuels were likely (even) less common in developing countries than today. A factor of 6 was used $^{2}$ to convert the weight of charcoal (metric tonnes) to the volume of wood $\left(\mathrm{m}^{3}\right)$ required to make the specified charcoal weight. It is unclear whether countries include the amount of wood used for charcoal making in the woodfuel statistics which they report to $\mathrm{FAO}^{3}$. To account for the fact that the data might be inconsistent, we used two methods (DIV1 and DIV2) to calculate

\footnotetext{
1 http://www.fao.org/faostat/en/\#data/F0, downloaded: 18 August 2018

2 http://www.fao.org/docrep/005/y4450e/y4450e13.htm, last access: 27 November 2018; a similar value of 7 is mentioned here: http://www.fao.org/docrep/x5667e/x5667e04.htm, last access: 17 Januar 2019

${ }^{3}$ http://www.fao.org/docrep/005/y4450e/y4450e13.htm
} 
the contribution of charcoal to wood fuel ( $\operatorname{frac}_{\text {DIV1 }}$ and $\left.\operatorname{frac}_{\text {DIV2 }}\right)$ :

$$
\operatorname{frac}_{\mathrm{DIV} 1}=\frac{\text { Wood }_{\mathrm{ch}}}{\text { Wood }_{\mathrm{con}}+\text { Wood }_{\text {noncon }}}
$$

and

$$
\operatorname{frac}_{\mathrm{DIV} 2}=\frac{\text { Wood }_{\mathrm{ch}}}{\text { Wood }_{\mathrm{ch}}+\text { Wood }_{\mathrm{con}}+\text { Wood }_{\text {noncon }}},
$$

where Wood $_{\mathrm{ch}}$ stands for the production of wood used for charcoal making, Wood ${ }_{\text {con }}$ for the production of coniferous fuel wood, and Wood $_{\text {noncon }}$ for the production of non-coniferous fuel wood. With method DIV1, we arrive at values above $100 \%$ for some countries. Since this makes no sense, we cap these values at $100 \%$.

We calculated the mean and the median fractions for both the world and Africa, the latter having the largest per capita use of charcoal in the developing world (Yevich and Logan, 2003). For both cases, we excluded all countries that do not produce charcoal, i.e. mainly developed countries that predominantly use fossil fuels. The values are shown in Table S7. The values lie in a relatively narrow range between $13 \%$ and $25 \%$. Although the large majority of people living in developing countries mainly used biomass for domestic energy (Wood and Baldwin, 1985), it is possible that the use of fossil fuels might affect our estimates to some degree.

Overall, the FAO data shows lower contributions of charcoal to total fuel wood than the estimates by Veal (2017). This could indicate that the estimates by Veal (2017) are too high for the whole Roman Empire (which was not the target of her study), since the Empire consisted of many parts that differed considerably with respect to wood supply. However, the Roman Empire had a high urbanisation rate $(\approx 10 \%$, with higher values in Italy; Temin, 2006$)$ and metallurgy was important (Harris, 2013), which speakes for a potentially higher contribution of charcoal than under present-day conditions. In the end, we decided to use a fraction of $50 \%$ of charcoal wood to total fuel wood, which is a compromise between the estimates by Veal (2017) and the FAO based values. To summarise, we assume that $20 \%$ of the fuel consisted of agricultural waste, $40 \%$ of charcoal (in terms of wood needed for charcoal production), and $40 \%$ of wood.

Tab. S3: Aerosol emission factors for wood burning $\left(E F_{\text {wood }}\right)$ in $\mathrm{g} \mathrm{kg}^{-1}$ used for the low, the intermediate, and the high scenarios.

\begin{tabular}{c|c|c|c}
\hline & Low estimate & Intermediate estimate & High estimate \\
\hline $\mathrm{BC}$ & 0.27 & 0.42 & 0.65 \\
$\mathrm{OC}$ & 0.84 & 2.09 & 4.15 \\
$\mathrm{SO}_{2}$ & 0.0021 & 0.098 & 0.20 \\
\hline
\end{tabular}

Tab. S4: Aerosol emission factors for agricultural waste burning (as fuel; $E F_{\text {agri }}$ ) in $\mathrm{g} \mathrm{kg}^{-1}$ used for the low, the intermediate, and the high scenarios.

\begin{tabular}{c|c|c|c}
\hline & Low estimate & Intermediate estimate & High estimate \\
\hline $\mathrm{BC}$ & 0.25 & 0.45 & 0.85 \\
$\mathrm{OC}$ & 1.16 & 2.40 & 4.32 \\
$\mathrm{SO}_{2}$ & 0.0082 & 0.022 & 0.050 \\
\hline
\end{tabular}


Tab. S5: Aerosol emission factors for charcoal burning $\left(E F_{\text {chb }}\right)$ in $\mathrm{g} \mathrm{kg}^{-1}$ (per kg charcoal) used for the low, the intermediate, and the high scenarios.

\begin{tabular}{c|r|r|r}
\hline & Low estimate & Intermediate estimate & High estimate \\
\hline $\mathrm{BC}$ & 0.44 & 0.59 & 0.79 \\
$\mathrm{OC}$ & 0.45 & 0.96 & 2.03 \\
$\mathrm{SO}_{2}$ & 0.29 & 0.43 & 0.62 \\
\hline
\end{tabular}

Tab. S6: Aerosol emission factors for charcoal production $\left(E F_{\text {chm }}\right)$ in $\mathrm{g} \mathrm{kg}^{-1}$ (per $\mathrm{kg}$ wood) used for the low, the intermediate, and the high scenarios.

\begin{tabular}{c|c|r|r}
\hline & Low estimate & Intermediate estimate & High estimate \\
\hline $\mathrm{BC}$ & 0.11 & 0.18 & 0.28 \\
$\mathrm{OC}$ & 3.21 & 3.81 & 4.51 \\
$\mathrm{SO}_{2}$ & 0.10 & 0.15 & 0.23 \\
\hline
\end{tabular}

Tab. S7: The percentages of wood used for charcoal making to total fuel wood. Two different methods (DIV1 and DIV2) were applied. Data was taken from FAO (year 1970).

\begin{tabular}{l|r|r|r|r}
\hline Method & World, mean & World, median & Africa, mean & Africa, median \\
\hline frac $_{\text {DIV1 }}$ & $25 \%$ & $16 \%$ & $24 \%$ & $16 \%$ \\
frac $_{\text {DIV2 }}$ & $18 \%$ & $13 \%$ & $18 \%$ & $14 \%$ \\
\hline
\end{tabular}

\section{S4 Estimating crop yield $Y$}

The agronomist Columella stated that the seed-yield ratios for most parts of Italy were seldom above 4:1 in the first century CE (Spurr, 1986). Assuming a sowing amount of $135 \mathrm{~kg} \mathrm{ha}^{-1}$ (advocated by the agronomists as a typical sowing amount; Goodchild, 2007), a ratio of 4:1 results in yields of $540 \mathrm{~kg} \mathrm{ha}^{-1}$. This yield is quite low compared to other sources, which show that yields of 5:1 and 6:1 were the most frequent in Italy and higher yields of 10-15:1 were not infrequent (Goodchild, 2007). For some regions, Varro (who lived in the first century BC) and Pliny the Elder (first century CE) reported even yields of 100:1 (Sinclair, 1998). So far, the estimates referred to Italy alone, but the variability between different Mediterranean countries was of course large: around 1921-1930 CE the average yields according to Hopkins (2017) ranged from values below $600 \mathrm{~kg} \mathrm{ha}^{-1}$ (Cyrenaica, Tunisia, Algeria), through values between 600 and $1000 \mathrm{~kg} \mathrm{ha}^{-1}$ (Greece, Portugal, Cyprus, Syria and Lebanon, Turkey, Spain) up to values above $1000 \mathrm{~kg} \mathrm{ha}^{-1}$ (Bulgaria, Yugoslavia, Italy, France, Egypt). Overall, we concluded that yields representative for the whole Roman Empire roughly lie in the range between $500 \mathrm{~kg} \mathrm{ha}^{-1}$ and $1000 \mathrm{~kg} \mathrm{ha}^{-1}$. 


\section{S5 Seasonal impact of anthropogenic land cover change}

Tab. S8: Absolute values of the reference simulation no human ("no") and the simulation LCC HYDE ("H") for each season (JJA=summer, $\mathrm{SON}=$ autumn, $\mathrm{DJF}=$ winter, and $\mathrm{MAM}=$ spring) and the whole year: cloud droplet number concentration burden, liquid water path, cloud cover, cloud radiative effect, wind velocity at $10 \mathrm{~m}$ altitude, surface albedo over land, evaporative fraction, and turbulent flux. The values are averaged from $10^{\circ} \mathrm{W}$ to $50^{\circ} \mathrm{E}, 20^{\circ} \mathrm{N}$ to $60^{\circ} \mathrm{N}$. Relative changes are shown in brackets and the stars indicate significant changes $(5 \%$ significance level; $N=20)$.

\begin{tabular}{|c|c|c|c|c|c|c|c|c|c|c|c|}
\hline Var. & Unit & no JJA & H JJA & no SON & $\mathrm{H} \mathrm{SON}$ & no DJF & H DJF & no MAM & H MAM & no year & H year \\
\hline CDNC & $10^{9} \mathrm{~m}^{-2}$ & 31.46 & $30.54(-2.9 \%)$ & 27.12 & $25.66 \quad(-5.4 \%)$ & 12.85 & $13.19 \quad(2.6 \%)$ & 22.49 & $21.92(-2.5 \%)$ & 23.52 & $22.87(-2.8 \%)$ \\
\hline LWP & $\mathrm{g} \mathrm{m}^{-2}$ & 54.96 & $54.89(-0.1 \%)$ & 59.03 & $(-4.5 \%)$ & 39.22 & $39.10(-0.3 \%)$ & 41.49 & $40.13(-3.3 \%)$ & 48.69 & $47.64(-2.2 \%)$ \\
\hline $\mathrm{CC}$ & - & 0.32 & $0.31(-3.1 \%)$ & 0.50 & $(2.1 \%)$ & 0.58 & $0.58 \quad(1.2 \%)$ & 0.54 & $0.54(-0.7 \%)$ & 0.48 & $0.48 \quad(0.2 \%)$ \\
\hline CRE & $\mathrm{W} \mathrm{m}^{-2}$ & -22.44 & $-22.58 \quad(0.6 \%)$ & -4.23 & $-2.91(-31.1 \%)$ & 4.57 & $(0.5 \%)$ & -14.43 & $-14.12(-2.1 \%)$ & -9.21 & $-8.83(-4.1 \%)$ \\
\hline Wind $_{10}$ & $\mathrm{~m} \mathrm{~s}^{-1}$ & 4.02 & $(0.8 \%)$ & 4.09 & $4.14 \quad(1.1 \%)$ & 4.63 & $(0.6 \%)$ & 4.33 & $4.37 \quad(1.1 \%)$ & 4.27 & $4.30 \quad(0.9 \%) *$ \\
\hline Albedo & - & 0.26 & $0.26 \quad(0.2 \%) *$ & 0.25 & $(-0.0 \%)$ & 0.26 & $0.26(-0.3 \%)$ & 0.25 & $0.25(-0.2 \%)$ & 0.26 & $0.26(-0.1 \%)$ \\
\hline Evap_frac & - & 0.36 & $0.36(-1.2 \%)$ & 0.32 & $(0.8 \%)$ & 0.32 & $0.33(3.2 \%) *$ & 0.43 & $0.43 \quad(0.7 \%)$ & 0.36 & $0.36 \quad(0.8 \%)$ \\
\hline $\mathrm{F}_{\text {turb }}$ & $\mathrm{W} \mathrm{m}^{-2}$ & 90.23 & $89.66(-0.6 \%)$ & 64.62 & $(0.3 \%)$ & 52.26 & $(0.9 \%)$ & 79.54 & $(0.5 \%)$ & 71.78 & $(0.2 \%)$ \\
\hline
\end{tabular}

Tab. S9: The same as Table $\mathrm{S} 8$ but for the simulations no_human and LCC_KK ("KK").

\begin{tabular}{|c|c|c|c|c|c|c|c|c|c|c|c|}
\hline Var. & Unit & no JJA & KK JJA & no SON & KK SON & no DJF & KK DJF & no MAM & KK MAM & no year & KK year \\
\hline CDNC & $10^{9} \mathrm{~m}^{-2}$ & 31.46 & $29.84(-5.2 \%)$ & 27.12 & $25.51 \quad(-5.9 \%)$ ** & 12.85 & $11.73(-8.7 \%)$ * * & 22.49 & $20.36(-9.5 \%)$ * * & 23.52 & $21.90(-6.9 \%) *$ \\
\hline LWP & $\mathrm{g} \mathrm{m}^{-2}$ & 54.96 & $53.48(-2.7 \%)$ & 59.03 & $(-5.2 \%)$ ** & 39.22 & $36.07(-8.1 \%)$ * * & 41.49 & $37.96(-8.5 \%) *$ & 48.69 & $45.89(-5.8 \%) *$ \\
\hline $\mathrm{CC}$ & - & 0.32 & $0.31(-1.7 \%)$ & 0.50 & $(1.3 \%)$ & 0.58 & $0.58 \quad(0.1 \%)$ & 0.54 & $0.53(-1.5 \%)$ & 0.48 & $0.48(-0.3 \%)$ \\
\hline CRE & $\mathrm{W} \mathrm{m}^{-2}$ & -22.44 & $-22.20(-1.1 \%)$ & -4.23 & $-3.13(-25.8 \%) *$ & 4.57 & $(0.4 \%)$ & -14.43 & $-13.28(-8.0 \%) *$ & -9.21 & $-8.58(-6.8 \%) *$ \\
\hline Wind $_{10}$ & $\mathrm{~m} \mathrm{~s}^{-1}$ & 4.02 & $4.18 \quad(3.9 \%)$ * & 4.09 & $4.22 \quad(3.2 \%)$ * & 4.63 & $(2.4 \%)$ * & 4.33 & $4.49 \quad(3.7 \%)$ * & 4.27 & $4.41 \quad(3.3 \%) *$ \\
\hline Albedo & - & 0.26 & $(0.8 \%) *$ & 0.25 & $(0.2 \%)$ & 0.26 & $(0.0 \%)$ & 0.25 & $0.25(-0.5 \%) *$ & 0.26 & $(0.1 \%)$ \\
\hline Evap_frac & - & 0.36 & $0.36 \quad(1.1 \%)$ & 0.32 & $(1.3 \%)$ & 0.32 & $(3.9 \%)$ 米 & 0.43 & $0.43 \quad(0.6 \%)$ & 0.36 & $(1.6 \%) *$ \\
\hline$F_{\text {turb }}$ & $\mathrm{W} \mathrm{m}{ }^{-2}$ & 90.23 & $90.20(-0.0 \%)$ & 64.62 & $(-0.8 \%)$ & 52.26 & $(0.1 \%)$ & 79.54 & $(1.7 \%)$ & 71.78 & $(0.3 \%)$ \\
\hline
\end{tabular}


S6 $\mathrm{OC}$ and $\mathrm{SO}_{2}$ emissions from anthropogenic sources and natural fire emissions around $100 \mathrm{CE}$

(a)

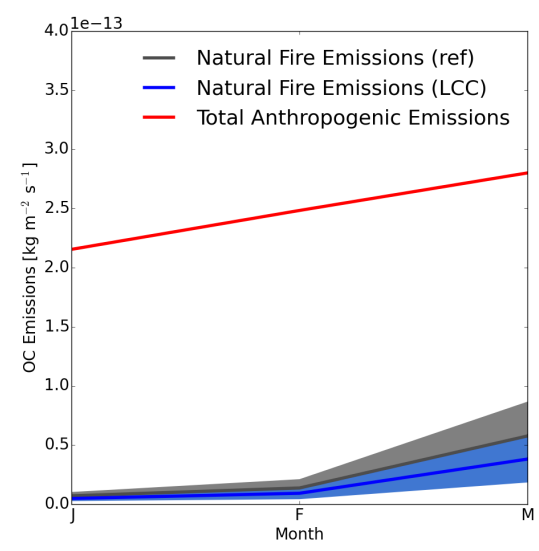

(c)

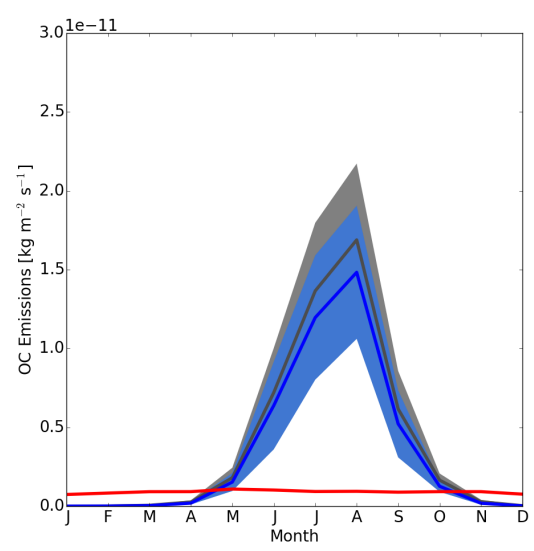

(b)

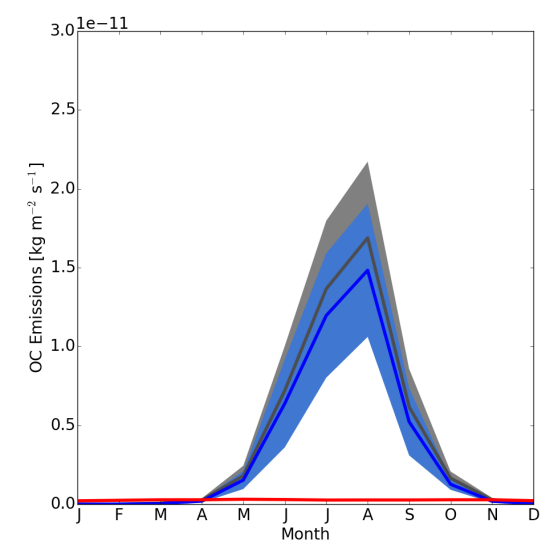

(d)

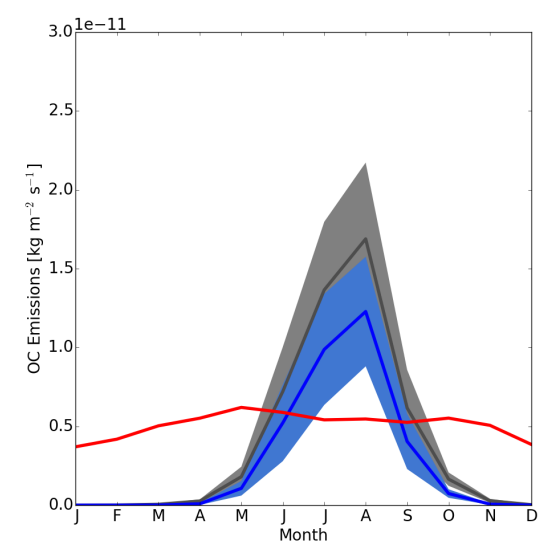

Fig. S2: The same as Fig. 4 in the manuscript but for OC instead of BC. 
(a)

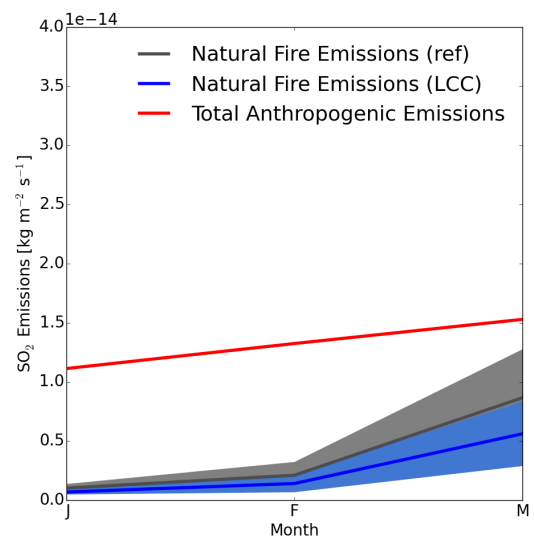

(c)

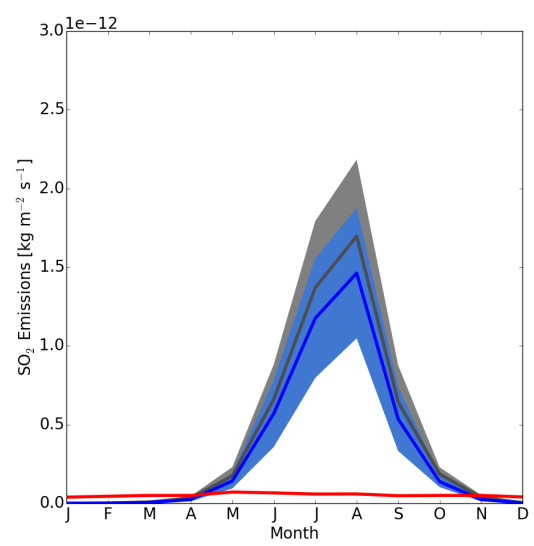

(b)

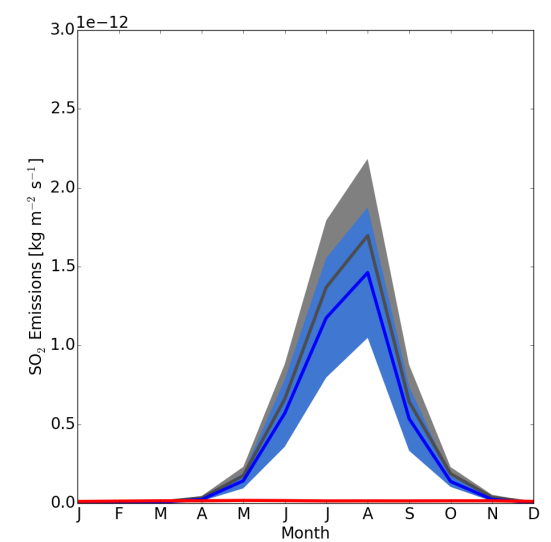

(d)

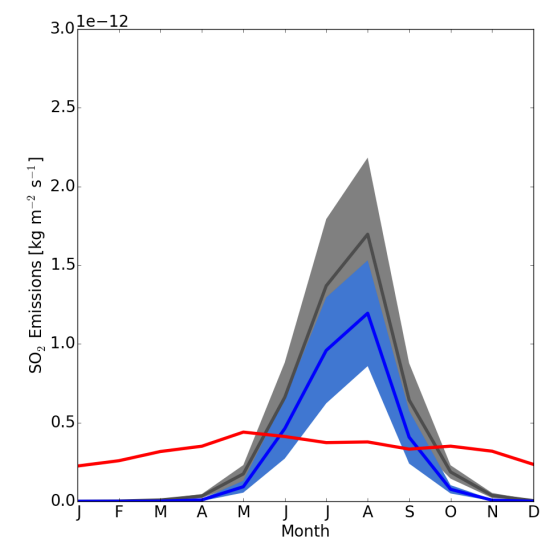

Fig. S3: The same as Fig. 4 in the manuscript but for $\mathrm{SO}_{2}$ instead of $\mathrm{BC}$. 
Tab. S11: The same as Table S10 but showing results for the simulations LCC_HYDE_int ("int") and LCC_HYDE.

\begin{tabular}{|c|c|c|c|c|c|c|c|c|c|c|c|}
\hline Var. & Unit & H JJA & int JJA & H SON & int SON & H DJF & int DJF & H MAM & int MAM & H year & int year \\
\hline $\mathrm{BC}$ burden & $\mu \mathrm{g} \mathrm{m}^{-2}$ & 538.37 & $454.27(-15.6 \%) *$ & 170.43 & $153.68(-9.8 \%)$ ** * & 2.72 & $14.93 \quad(448.8 \%) *$ & 26.46 & $44.12 \quad(66.7 \%)$ * * & 185.42 & $167.54 \quad(-9.6 \%) *$ \\
\hline OM burden & $\mu \mathrm{g} \mathrm{m}^{-2}$ & 10151.91 & $8781.79(-13.5 \%)$ * * & 3320.51 & $3180.92 \quad(-4.2 \%)$ & 677.58 & $(25.5 \%) *$ & 1242.32 & $(22.0 \%) *$ & 3865.01 & $(-6.9 \%) *$ \\
\hline $\mathrm{SO}_{4}$ burden & $\mu \mathrm{g} \mathrm{m} \mathrm{m}^{-2}$ & 6331.80 & $6067.79(-4.2 \%)$ & 4569.16 & 4613.68 & 2600.15 & 2682.92 & 3733.66 & $(-1.4 \%)$ & 4316.42 & $(-1.1 \%)$ \\
\hline CDNC & $10^{9} \mathrm{~m}^{-2}$ & 30.54 & $(13.3 \%) *$ & 25.66 & $(41.7 \%) *$ & 13.19 & $(121.2 \%) *$ & 21.92 & $(40.8 \%) *$ & 22.87 & $(43.2 \%) *$ \\
\hline LWP & $\mathrm{g} \mathrm{m}^{-2}$ & 54.89 & $(7.4 \%) *$ & 56.36 & $(35.2 \%) *$ & 39.10 & $(98.1 \%)$ * * & 40.13 & $(38.5 \%)$ * & 47.64 & $(40.6 \%) *$ \\
\hline $\mathrm{CC}$ & - & 0.31 & $(3.8 \%)$ & 0.51 & $(3.9 \%)$ & 0.58 & $(6.1 \%) *$ & 0.54 & $(2.7 \%) *$ & 0.48 & $(4.2 \%) *$ \\
\hline ARE & $\mathrm{W} \mathrm{m}^{-2}$ & -0.81 & $(-2.8 \%)$ & -0.94 & $-0.92 \quad(-2.1 \%)$ & -0.51 & $-0.43 \quad(-16.3 \%)$ * * & -0.40 & $-0.05(-88.6 \%) *$ & -0.66 & $-0.54(-18.0 \%) *$ \\
\hline CRE & $\mathrm{W} \mathrm{m}^{-2}$ & -22.58 & $(6.9 \%) *$ & -2.91 & $-5.96(104.7 \%) *$ & 4.60 & $-0.86(-118.7 \%)$ * * & -14.12 & $-20.77 \quad(47.1 \%)$ * * & -8.83 & $-13.01 \quad(47.2 \%) *$ \\
\hline
\end{tabular}


Tab. S12: The same as Table $\mathrm{S} 10$ but showing results for LCC_KK and LCC_KK_high ("high").

\begin{tabular}{|c|c|c|c|c|c|c|c|c|c|c|c|c|c|c|}
\hline Var. & Unit & KK JJA & high JJA & KK SON & high & $1 \mathrm{SON}$ & KK DJF & hig & h DJF & KK MAM & high & MAM & KK year & high year \\
\hline BC burden & $\mu g \mathrm{~m}^{-2}$ & 516.73 & $520.93 \quad(0.8 \%)$ & 169.10 & 233.65 & $(38.2 \%) *$ & 2.67 & 70.53 & $(2542.2 \%)$ * & 26.37 & 131.05 & $(397.0 \%)$ * & 179.61 & $239.89 \quad(33.6 \%) *$ \\
\hline OM burden & $\mu \mathrm{g} \mathrm{m}^{-2}$ & 9466.62 & 9534.91 & 3210.24 & 4332.61 & $(35.0 \%) *$ & 623.35 & 1600.17 & $(156.7 \%) *$ & 1145.62 & 2634.95 & $(130.0 \%) *$ & 3627.28 & $(25.2 \%) *$ \\
\hline $\mathrm{SO}_{4}$ burden & $\mu \mathrm{g} \mathrm{m}^{-2}$ & 6280.78 & $6065.80(-3.4 \%)$ & 4646.45 & 4765.02 & $(2.6 \%)$ & 2566.28 & 2820.00 & $(9.9 \%)$ * & 3699.46 & 3729.81 & $(0.8 \%)$ & 4305.81 & 4351.47 \\
\hline CDNC & $10^{9} \mathrm{~m}^{-2}$ & 29.84 & 42.75 (43.3\%)* & 25.51 & 62.60 & $(145.4 \%) *$ & 11.73 & 67.39 & $(474.5 \%) *$ & 20.36 & 60.85 & $(198.8 \%) * *$ & 21.90 & $58.34 \quad(166.4 \%) *$ \\
\hline LWP & $\mathrm{g} \mathrm{m}^{-2}$ & 53.48 & $(18.2 \%) *$ & 55.95 & 91.92 & $(64.3 \%) *$ & 36.07 & 106.09 & $(194.2 \%) *$ & 37.96 & 77.16 & $(103.3 \%)$ * & 45.89 & $(84.1 \%) *$ \\
\hline $\mathrm{CC}$ & - & 0.31 & $(6.6 \%) *$ & 0.50 & 0.54 & $(7.6 \%) *$ & 0.58 & 0.64 & $(10.7 \%)$ 米 & 0.53 & 0.58 & (8.8\%)* * & 0.48 & $(8.7 \%) *$ \\
\hline $\mathrm{ARE}$ & $\mathrm{W} \mathrm{m}^{-2}$ & -0.68 & $-0.81 \quad(19.9 \%)$ & -1.00 & -0.97 & $(-2.9 \%)$ & -0.51 & -0.40 & $(-21.5 \%) *$ & -0.37 & 0.10 & $(-127.3 \%) *$ & -0.64 & $-0.52(-18.7 \%) *$ \\
\hline $\mathrm{CRE}$ & $\mathrm{W} \mathrm{m}^{-2}$ & -22.20 & $(13.9 \%) *$ & -3.13 & -7.21 & $(130.0 \%) *$ & 4.59 & -4.33 & $(-194.3 \%)$ * & -13.28 & -27.69 & $(108.5 \%)$ 州 & -8.58 & $-16.21 \quad(88.9 \%) *$ \\
\hline
\end{tabular}




\section{S8 Fixed SSTs versus MLO}

(a)

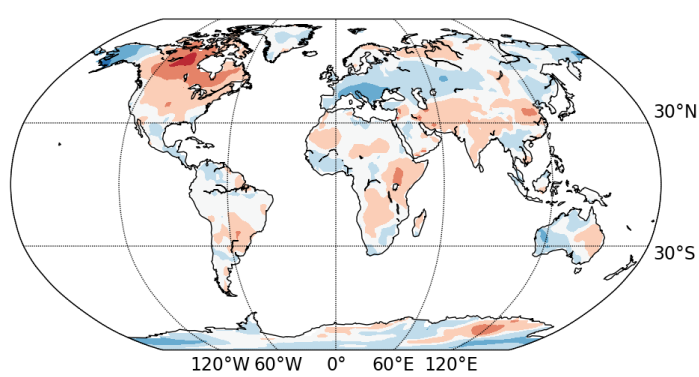

(b)

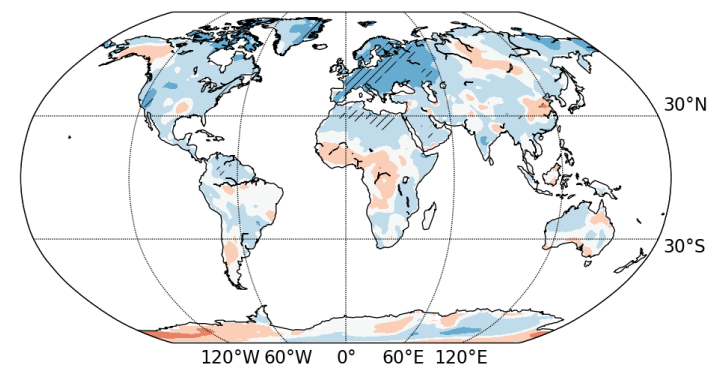

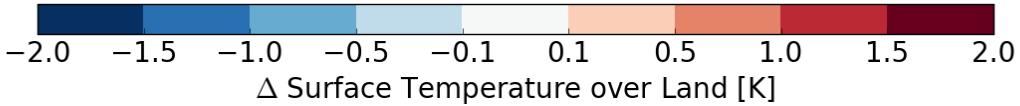

Fig. S4: The impact of anthropogenic land cover and aerosol emissions on land surface temperature for the intermediate emission scenario (difference between LCC_HYDE_int and no_human). Shown are changes simulated with fixed SSTs (a) and changes simulated with an MLO (b).

\section{S9 Comparison between CBALONE-SPITFIRE emissions and emissions by van Marle et al. (2017)}

Here, we compare the CBALONE-SPITFIRE emissions around $1835 \mathrm{CE}$ with the emissions of van Marle et al. (2017) around $1835 \mathrm{CE}$, which could show biases in the fire model. The reconstructed emissions by van Marle et al. (2017) are used as input for simulations in the context of the Climate Model Intercomparison Project phase 6; we therefore call them "CMIP6 emissions" in the following. The CMIP6 emissions were calculated by merging satellite data with proxies for biomass burning (e.g. charcoal records and visibility observations) next to using the average of six fire models. The emissions simulated by CBALONE-SPITFIRE are not totally independent of the CMIP6 emissions because 3 of the 6 models considered by van Marle et al. (2017) include SPITFIRE (one of them was JSBACH-SPITFIRE).

The global averages of the fire emissions are relatively similar between CBALONE-SPITFIRE and CMIP6 around $1835 \mathrm{CE}$ (Table $\mathrm{S} 13$ ). The values are somewhat larger for CBALONESPITFIRE, namely a factor of 1.2 for $\mathrm{SO}_{2}$ and a factor of 1.7 for $\mathrm{BC}$ and $\mathrm{OC}$ emissions. However, one should keep in mind that the fire emissions by CMIP6 are not the truth and also uncertain. For example, Hamilton et al. (2018) concluded that the CMIP6 pre-industrial fire emissions are likely underestimated.

Although global averages are similar, CBALONE-SPITFIRE simulates pronouncedly higher aerosol emissions compared to CMIP6 in our study domain around 1835 CE (Tables S14, S15, S16). The disagreement is larger in summer and autumn (CBALONE-SPITFIRE 7 to 15 times higher) than in winter and spring (CBALONE-SPITFIRE 2 to 4 times higher). Furthermore, the fire emissions in winter are more located to the South in CBALONE-SPITFIRE; it simulates basically no fires in Eastern Europe in contrast to CMIP6, but higher emissions in North Africa and the Middle East (not shown). Note that the majority ( $\approx 80 \%$ in $1850 \mathrm{CE}$ ) of the 
CMIP6 fire emissions in the study domain originates from agricultural crop waste burning on field, whereas crop area is excluded from burning in CBALONE-SPITFIRE. The CBALONESPITFIRE emissions could differ from the CMIP6 emissions over Europe because the first are only model-based, while the latter also include observational data. Furthermore, the models considered by van Marle et al. (2017) were driven by different forcing data than our CBALONESPITFIRE simulations.

Overall, the fire emissions calculated with CBALONE-SPITFIRE are higher than the CMIP6 emissions in the study domain around $1835 \mathrm{CE}$. As a consequence, the natural fire emissions calculated for $100 \mathrm{CE}$ could be overestimated in the study domain.

Tab. S13: Global mean fire emissions (in $10^{-14} \mathrm{~kg} \mathrm{~m}^{-2} \mathrm{~s}^{-1}$ ) around $1835 \mathrm{CE}$ used for CMIP6 and those calculated with CBALONE-SPITFIRE ("CBAL-SPIT"). The data covers 20 years around $1835 \mathrm{CE}(1823-1842 \mathrm{CE})$.

\begin{tabular}{l|c|c}
\hline & CMIP6 & CBAL-SPIT \\
\hline $\mathrm{BC}$ & 10.0 & 17.0 \\
$\mathrm{OC}$ & 84.6 & 141 \\
$\mathrm{SO}_{2}$ & 12.5 & 14.5 \\
\hline
\end{tabular}

Tab. S14: Mean BC fire emissions (in $10^{-14} \mathrm{~kg} \mathrm{~m}^{-2} \mathrm{~s}^{-1}$ ) averaged from $10^{\circ} \mathrm{W}$ to $50^{\circ} \mathrm{E}, 20^{\circ} \mathrm{N}$ to $60^{\circ} \mathrm{N}$ used for CMIP6 and those calculated with CBALONE-SPITFIRE ("CBALSPIT"). The data covers 20 years around $1835 \mathrm{CE}$ (1823-1842 CE).

\begin{tabular}{l|c|c|c}
\hline & CMIP6 1835 CE & CBAL-SPIT 1835 CE & CBAL-SPIT 100 CE \\
\hline Autumn & 6.65 & 62.4 & 33.9 \\
Winter & 0.470 & 1.95 & 0.339 \\
Spring & 6.29 & 16.7 & 9.20 \\
Summer & 14.8 & 135 & 142 \\
\hline
\end{tabular}

Tab. S15: The same as Table S14 but for OC and in $10^{-13} \mathrm{~kg} \mathrm{~m}^{-2} \mathrm{~s}^{-1}$.

\begin{tabular}{l|r|r|c}
\hline & CMIP6 1835 CE & CBAL-SPIT 1835 CE & CBAL-SPIT 100 CE \\
\hline Autumn & 2.80 & 43.0 & 26.9 \\
Winter & 0.295 & 1.07 & 0.178 \\
Spring & 3.39 & 11.1 & 7.14 \\
Summer & 6.77 & 101 & 126 \\
\hline
\end{tabular}

Tab. S16: The same as Table $\mathrm{S} 14$ but for $\mathrm{SO}_{2}$ (in $\left.10^{-14} \mathrm{~kg} \mathrm{~m}^{-2} \mathrm{~s}^{-1}\right)$.

\begin{tabular}{l|r|r|c}
\hline & CMIP6 1835 CE & CBAL-SPIT 1835 CE & CBAL-SPIT 100 CE \\
\hline Autumn & 4.54 & 40.2 & 28.8 \\
Winter & 0.435 & 1.57 & 0.250 \\
Spring & 5.06 & 10.2 & 7.27 \\
Summer & 10.8 & 78.7 & 125 \\
\hline
\end{tabular}

Comparing the two CBALONE-SPITFIRE simulations (1835 CE versus $100 \mathrm{CE}$; Tables S14, S15. S16) reveals that emissions around $1835 \mathrm{CE}$ were somewhat lower in summer compared to $100 \mathrm{CE}$. However, for the other seasons, the emissions in $1835 \mathrm{CE}$ are higher, especially in winter (a factor of 6). The differences can partly be explained by the differences in the population density; for $100 \mathrm{CE}$, only natural fires were calculated and the population density was thus set to 0 (Sect. 2.4). In the model, population shortens the fire duration but at the 
same time increases the number of ignition events. While the first effect dominates in summer, the second one dominates in the other seasons. Furthermore, differences in anthropogenic land cover (none assumed for $100 \mathrm{CE}$ ), natural vegetation, and climate can also contribute to the differences between $1835 \mathrm{CE}$ and $100 \mathrm{CE}$. 


\begin{tabular}{|c|c|c|c|c|c|c|c|c|c|c|}
\hline Burning material & $\mathrm{OC}$ & & $\mathrm{BC}$ & & $\mathrm{SO}_{2}$ & & $N$ & Reference & Burning/ measurement device & Key \\
\hline & average & std & average & std & average & std & & & & \\
\hline UP dung & 7.1970 & 6.7530 & 0.5657 & 0.4949 & & & 7 & $\begin{array}{l}\text { Pandey et al. } \\
(2017)\end{array}$ & $\begin{array}{l}\text { traditional cooking-stove, In- } \\
\text { dia }\end{array}$ & 2 \\
\hline Bihar dung & 10.6500 & 10.0300 & 0.9700 & 0.8403 & & & 6 & $\begin{array}{l}\text { Pandey et al. } \\
(2017)\end{array}$ & $\begin{array}{l}\text { traditional cooking-stove, In- } \\
\text { dia }\end{array}$ & 2 \\
\hline Chh rice straw & 7.0786 & 5.1236 & 1.1157 & 1.3508 & & & 7 & $\begin{array}{l}\text { Pandey et al. } \\
(2017)\end{array}$ & $\begin{array}{l}\text { traditional cooking-stove, In- } \\
\text { dia }\end{array}$ & 2 \\
\hline Chh tur stalk & 8.0880 & 6.1330 & 1.9580 & 1.8140 & & & 10 & $\begin{array}{l}\text { Pandey et al. } \\
(2017)\end{array}$ & $\begin{array}{l}\text { traditional cooking-stove, In- } \\
\text { dia }\end{array}$ & 2 \\
\hline Punjab wood & 2.6000 & 2.3780 & 0.6750 & 0.4387 & & & 4 & $\begin{array}{l}\text { Pandey et al. } \\
(2017)\end{array}$ & $\begin{array}{l}\text { traditional cooking-stove, In- } \\
\text { dia }\end{array}$ & 1 \\
\hline Raj wood & 5.4041 & 5.0848 & 1.1410 & 1.0134 & & & 17 & $\begin{array}{l}\text { Pandey et al. } \\
(2017)\end{array}$ & $\begin{array}{l}\text { traditional cooking-stove, In- } \\
\text { dia }\end{array}$ & 1 \\
\hline UP wood & 2.1363 & 1.1830 & 0.5500 & 0.3464 & & & 8 & $\begin{array}{l}\text { Pandey et al. } \\
(2017)\end{array}$ & $\begin{array}{l}\text { traditional cooking-stove, In- } \\
\text { dia }\end{array}$ & 1 \\
\hline AP wood & 5.0125 & 3.6248 & 0.4550 & 0.2664 & & & 4 & $\begin{array}{l}\text { Pandey et al. } \\
(2017)\end{array}$ & $\begin{array}{l}\text { traditional cooking-stove, In- } \\
\text { dia }\end{array}$ & 1 \\
\hline wheat straw & 3.4600 & 2.0500 & 0.4200 & 0.2300 & 0.0400 & 0.0400 & 8 & Cao et al. (2008) & $\begin{array}{l}\text { combustion tower (simulating } \\
\text { cooking in traditional stoves) }\end{array}$ & 2 \\
\hline rice straw & 2.0100 & 0.6700 & 0.4900 & 0.2100 & 0.1800 & 0.3100 & 14 & Cao et al. (2008) & $\begin{array}{l}\text { combustion tower (simulating } \\
\text { cooking in traditional stoves) }\end{array}$ & 2 \\
\hline
\end{tabular}




\begin{tabular}{|c|c|c|c|c|c|c|c|c|c|c|}
\hline cotton stalk & 1.8300 & 0.5400 & 0.8200 & 0.2000 & 0.0400 & 0.0400 & 2 & $\begin{array}{l}\text { Cao et al. } 2008) \\
\text { Cao et al. }(2008)\end{array}$ & $\begin{array}{l}\text { combustion tower (simulating } \\
\text { cooking in traditional stoves) } \\
\text { combustion tower (simulating } \\
\text { cooking in traditional stoves) }\end{array}$ & 2 \\
\hline rice straw & 0.9000 & 0.4500 & 0.4600 & 0.2300 & & & 7 & Turn et al. (1997) & wind tunnel & $3 \mathrm{a}$ \\
\hline wheat straw & 2.1000 & 1.0500 & 0.7900 & 0.3950 & & & 2 & Turn et al. (1997) & wind tunnel & $3 \mathrm{a}$ \\
\hline barley straw & 3.0000 & 1.5000 & 1.2000 & 0.6000 & & & 2 & Turn et al. (1997) & wind tunnel & $3 \mathrm{a}$ \\
\hline corn stover & 1.7000 & 0.8500 & 0.6700 & 0.3350 & & & 2 & Turn et al. (1997) & wind tunnel & $3 \mathrm{a}$ \\
\hline sugar cane & 1.5000 & 0.7500 & 0.5900 & 0.2950 & & & 3 & Turn et al. (1997) & wind tunnel & $3 \mathrm{a}$ \\
\hline wheat straw & 2.8130 & 0.1470 & 0.6760 & 0.0270 & & & 4 & Li et al. $(2017)$ & $\begin{array}{l}\text { combustion stove to simulate } \\
\text { open burning }\end{array}$ & 3 \\
\hline corn straw & 2.3930 & 0.3510 & 0.7780 & 0.1520 & & & 4 & Li et al. (2017) & $\begin{array}{l}\text { combustion stove to simulate } \\
\text { open burning }\end{array}$ & 3 \\
\hline rice straw & 6.8820 & 0.6890 & 2.1820 & 0.2780 & & & 4 & Li et al. (2017) & $\begin{array}{l}\text { combustion stove to simulate } \\
\text { open burning }\end{array}$ & 3 \\
\hline cotton residue & 7.4150 & 0.5470 & 1.1920 & 0.1710 & & & 4 & Li et al. (2017) & $\begin{array}{l}\text { combustion stove to simulate } \\
\text { open burning }\end{array}$ & 3 \\
\hline soybean residue & 1.5390 & 0.2530 & 0.6140 & 0.1900 & & & 4 & Li et al. $(2017)$ & $\begin{array}{l}\text { combustion stove to simulate } \\
\text { open burning }\end{array}$ & 3 \\
\hline Maize-brick & & & & & 0.0163 & 0.0104 & 3 & $\begin{array}{l}\text { Zhang et al. } \\
(2000)\end{array}$ & brick stove with flue & 2 \\
\hline Wood-India & & & & & 0.0024 & 0.0024 & 3 & Zhang et al. & $\begin{array}{l}\text { metal stove without flue (from } \\
\text { India) }\end{array}$ & 1 \\
\hline Wheat-brick & & & & & 0.0335 & 0.0174 & 3 & Zhang et al. & brick stove with flue & 2 \\
\hline Brush-brick & & & & & 0.0056 & 0.0077 & 3 & Zhang et al. & brick stove with flue & 2 \\
\hline Brush-India & & & & & 0.0025 & 0.0044 & 3 & Zhang et al. & $\begin{array}{l}\text { metal stove without flue (from } \\
\text { India) }\end{array}$ & 2 \\
\hline
\end{tabular}




\begin{tabular}{|c|c|c|c|c|c|c|c|c|}
\hline $\begin{array}{l}\text { fireplace, soft- } \\
\text { wood }\end{array}$ & 3.0073 & 0.3335 & 0.7742 & 0.0697 & 3 & $\begin{array}{l}\text { McDonald et al. } \\
(2000)\end{array}$ & heatilator model E36 fireplace & 1 \\
\hline $\begin{array}{l}\text { fireplace, hard- } \\
\text { wood }\end{array}$ & 3.5800 & 0.7981 & 0.3975 & 0.1177 & 3 & $\begin{array}{l}\text { McDonald et al. } \\
(2000)\end{array}$ & heatilator model E36 fireplace & 1 \\
\hline $\begin{array}{l}\text { wood stove, hard- } \\
\text { wood }\end{array}$ & 2.8211 & 1.7706 & 0.3563 & 0.2169 & 3 & McDonald et al. & $\begin{array}{l}\text { noncatalytic Pineridge appli- } \\
\text { cance }\end{array}$ & 1 \\
\hline maritime pine & 6.1344 & 3.0672 & 1.0650 & 0.5325 & 3 & Alves et al. (2011) & $\begin{array}{l}\text { traditional Portuguese brick } \\
\text { open fireplace }\end{array}$ & $1 \mathrm{a}$ \\
\hline eucalypt & 8.7264 & 4.3632 & 0.4444 & 0.2222 & 3 & Alves et al. (2011) & $\begin{array}{l}\text { traditional Portuguese brick } \\
\text { open fireplace }\end{array}$ & $1 \mathrm{a}$ \\
\hline cork oak & 6.9412 & 3.4706 & 0.3484 & 0.1742 & 3 & Alves et al. (2011) & $\begin{array}{l}\text { traditional Portuguese brick } \\
\text { open fireplace }\end{array}$ & $1 \mathrm{a}$ \\
\hline golden wattle & 3.8700 & 1.9350 & 0.6600 & 0.3300 & 3 & Alves et al. (2011) & $\begin{array}{l}\text { traditional Portuguese brick } \\
\text { open fireplace }\end{array}$ & $1 \mathrm{a}$ \\
\hline olive & 4.8015 & 2.4008 & 0.4158 & 0.2079 & 3 & Alves et al. (2011) & $\begin{array}{l}\text { traditional Portuguese brick } \\
\text { open fireplace }\end{array}$ & $1 \mathrm{a}$ \\
\hline Portugese oak & 9.1680 & 4.5840 & 0.4775 & 0.2388 & 3 & Alves et al. (2011) & $\begin{array}{l}\text { traditional Portuguese brick } \\
\text { open fireplace }\end{array}$ & $1 \mathrm{a}$ \\
\hline holm oak & 8.4800 & 4.2400 & 0.3520 & 0.1760 & 3 & Alves et al. (2011) & $\begin{array}{l}\text { traditional Portuguese brick } \\
\text { open fireplace }\end{array}$ & $1 \mathrm{a}$ \\
\hline maritime pine & 8.0196 & 4.0098 & 0.6357 & 0.3179 & 3 & Alves et al. (2011) & $\begin{array}{l}\text { a cast iron woodstove (Solza- } \\
\text { ima, model Sahara) }\end{array}$ & $1 \mathrm{a}$ \\
\hline eucalypt & 3.2160 & 1.6080 & 0.2479 & 0.1240 & 3 & Alves et al. (2011) & $\begin{array}{l}\text { a cast iron woodstove (Solza- } \\
\text { ima, model Sahara) }\end{array}$ & $1 \mathrm{a}$ \\
\hline cork oak & 8.0936 & 4.0468 & 0.2869 & 0.1435 & 3 & Alves et al. (2011) & $\begin{array}{l}\text { a cast iron woodstove (Solza- } \\
\text { ima, model Sahara) }\end{array}$ & $1 \mathrm{a}$ \\
\hline golden wattle & 5.2650 & 2.6325 & 0.2340 & 0.1170 & 3 & Alves et al. (2011) & $\begin{array}{l}\text { a cast iron woodstove (Solza- } \\
\text { ima, model Sahara) }\end{array}$ & $1 \mathrm{a}$ \\
\hline
\end{tabular}




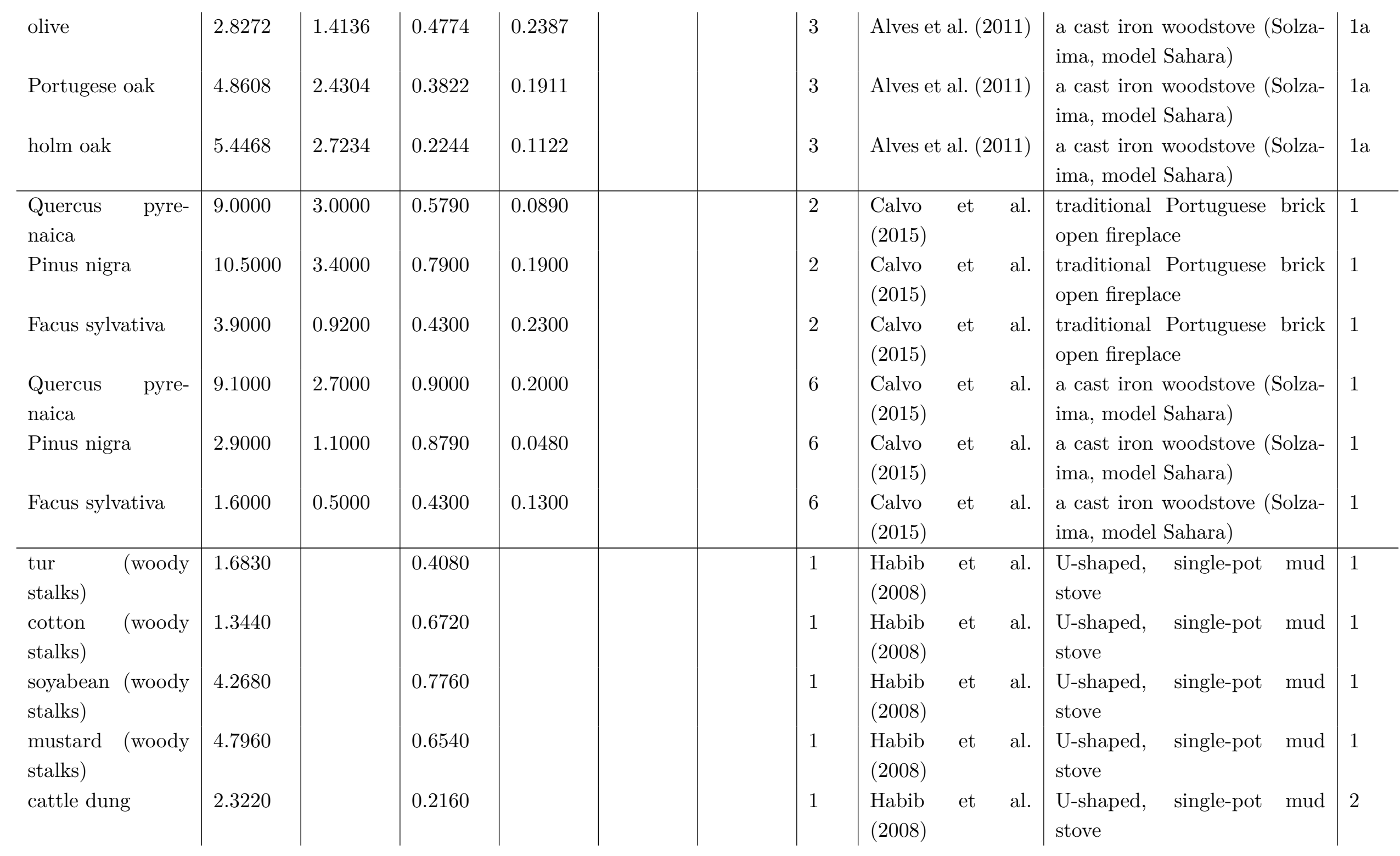




\begin{tabular}{|c|c|c|c|c|c|c|c|c|}
\hline \multirow{2}{*}{$\begin{array}{l}\text { jute (fibrous hol- } \\
\text { low stalks) } \\
\text { rice straw }\end{array}$} & 0.6290 & & 0.3060 & & 1 & Habib et al. & $\begin{array}{l}\text { U-shaped, single-pot mud } \\
\text { stove }\end{array}$ & 2 \\
\hline & 4.6500 & & 0.1860 & & 1 & $\begin{array}{l}\text { Habib et al. } \\
(2008)\end{array}$ & $\begin{array}{l}\text { U-shaped, single-pot mud } \\
\text { stove }\end{array}$ & 2 \\
\hline jamun & 1.5800 & 2.0300 & 0.4800 & 0.0850 & 2 & $\begin{array}{l}\text { Habib et al. } \\
(2008)\end{array}$ & $\begin{array}{l}\text { U-shaped, single-pot mud } \\
\text { stove }\end{array}$ & 1 \\
\hline neem & 1.6500 & 1.9500 & 0.6100 & 0.0300 & 2 & $\begin{array}{l}\text { Habib et al. } \\
(2008)\end{array}$ & $\begin{array}{l}\text { U-shaped, single-pot mud } \\
\text { stove }\end{array}$ & 1 \\
\hline mango & 1.6100 & 1.5700 & 0.6400 & 0.0640 & 2 & $\begin{array}{c}\text { Habib et al. } \\
(2008)\end{array}$ & $\begin{array}{l}\text { U-shaped, single-pot mud } \\
\text { stove }\end{array}$ & 1 \\
\hline Acacia & 1.1000 & 0.5390 & 0.4500 & 0.1220 & 2 & $\begin{array}{l}\text { Habib } \\
(2008)\end{array}$ & $\begin{array}{l}\text { U-shaped, single-pot mud } \\
\text { stove }\end{array}$ & 1 \\
\hline horsebean & 1.2000 & 0.1700 & 1.2800 & 0.1000 & 2 & Shen et al. $(2010)$ & brick wok stove (rural) & 2 \\
\hline peanut & 1.0800 & & 0.4930 & & 1 & Shen et al. (2010) & brick wok stove (rural) & 2 \\
\hline soybean residue & 1.1700 & 0.1800 & 1.3700 & 0.0400 & 2 & Shen et al. (2010) & brick wok stove (rural) & 2 \\
\hline cotton residue & 0.3540 & 0.0410 & 1.3400 & 0.5800 & 2 & Shen et al. $(2010)$ & brick wok stove (rural) & 2 \\
\hline rice & 1.5000 & 0.1600 & 0.7490 & 0.4270 & 2 & Shen et al. (2010) & brick wok stove (rural) & 2 \\
\hline wheat & 2.2700 & 1.3000 & 2.6400 & 1.0100 & 2 & Shen et al. (2010) & brick wok stove (rural) & 2 \\
\hline rape & 1.7500 & 0.6300 & 2.3400 & 0.9200 & 2 & Shen et al. $(2010)$ & brick wok stove (rural) & 2 \\
\hline sesame & 2.3400 & 1.1200 & 1.0700 & 0.0300 & 2 & Shen et al. (2010) & brick wok stove (rural) & 2 \\
\hline corn & 1.3600 & 0.2900 & 1.1100 & 0.0800 & 2 & Shen et al. $(2010)$ & brick wok stove (rural) & 2 \\
\hline wheat stubble & 1.3794 & 0.3012 & 0.4260 & 0.1147 & 3 & $\begin{array}{l}\text { Jimenez et al. } \\
(2007)\end{array}$ & $\begin{array}{l}\text { test burn chamber to mimic } \\
\text { open burn conditions }\end{array}$ & 3 \\
\hline wheat stubble & 2.8120 & 1.6127 & 0.1660 & 0.1408 & 6 & $\begin{array}{l}\text { Jimenez et al. } \\
(2007)\end{array}$ & open field & 3 \\
\hline $\begin{array}{l}\text { Kentucky Blue- } \\
\text { grass }\end{array}$ & 5.2248 & 2.2406 & 0.7650 & 0.3361 & 2 & $\begin{array}{l}\text { Jimenez et al. } \\
(2007)\end{array}$ & open field & 4 \\
\hline $\begin{array}{l}\text { Kentucky Blue- } \\
\text { grass }\end{array}$ & 4.4637 & 1.2962 & 0.8155 & 0.4307 & 4 & $\begin{array}{l}\text { Jimenez et al. } \\
(2007)\end{array}$ & $\begin{array}{l}\text { test burn chamber to mimic } \\
\text { open burn conditions }\end{array}$ & 4 \\
\hline
\end{tabular}




\begin{tabular}{|c|c|c|c|c|c|c|c|c|}
\hline wheat straw & 0.2900 & 0.1200 & 0.1600 & 0.0700 & & Sahai et al. (2007) & field burning & 3 \\
\hline wheat straw & 2.3800 & & 1.5900 & & 1 & Sahai et al. $(2007)$ & $\begin{array}{l}\text { earlier lab experiments at } \\
\text { NPL }\end{array}$ & 3 \\
\hline wheat straw & 1.2300 & & 0.5200 & & 1 & Hays et al. (2005) & field open burn simulations & 3 \\
\hline rice straw & 8.9400 & & 0.1700 & & 1 & Hays et al. (2005) & field open burn simulations & 3 \\
\hline olive pits & 0.9000 & 0.5700 & 0.1000 & 0.0600 & & $\begin{array}{l}\text { AIRUSE LIFE } \\
(2016)\end{array}$ & from pellet stove & 2 \\
\hline maritime pine & 2.5400 & 2.5100 & 0.6100 & 0.4300 & 3 & Fernandes et al. & $\begin{array}{l}\text { a cast iron woodstove (Solza- } \\
\text { ima, model Sahara) }\end{array}$ & 1 \\
\hline golden wattle & 4.0700 & 2.6500 & 0.2900 & 0.1800 & 3 & Fernandes et al. & $\begin{array}{l}\text { a cast iron woodstove (Solza- } \\
\text { ima, model Sahara) }\end{array}$ & 1 \\
\hline eucalypt & 5.1600 & 4.0300 & 0.3700 & 0.3000 & 3 & $\begin{array}{l}\text { Fernandes et al. } \\
(2011)\end{array}$ & $\begin{array}{l}\text { a cast iron woodstove (Solza- } \\
\text { ima, model Sahara) }\end{array}$ & 1 \\
\hline cork oak & 4.8000 & 3.3800 & 0.4200 & 0.3300 & 3 & $\begin{array}{l}\text { Fernandes et al. } \\
(2011)\end{array}$ & $\begin{array}{l}\text { a cast iron woodstove (Solza- } \\
\text { ima, model Sahara) }\end{array}$ & 1 \\
\hline olive & 4.5500 & 2.2200 & 0.4600 & 0.2400 & 3 & Fernandes et al. & $\begin{array}{l}\text { a cast iron woodstove (Solza- } \\
\text { ima, model Sahara) }\end{array}$ & 1 \\
\hline holm oak & 3.0300 & 2.0500 & 0.2300 & 0.0900 & 3 & $\begin{array}{l}\text { Fernandes et al. } \\
(2011)\end{array}$ & $\begin{array}{l}\text { a cast iron woodstove (Solza- } \\
\text { ima, model Sahara) }\end{array}$ & 1 \\
\hline Portugese oak & 6.1700 & 4.6200 & 0.3200 & 0.1500 & 3 & $\begin{array}{l}\text { Fernandes et al. } \\
(2011)\end{array}$ & $\begin{array}{l}\text { a cast iron woodstove (Solza- } \\
\text { ima, model Sahara) }\end{array}$ & 1 \\
\hline maritime pine & 2.9100 & 1.3000 & 0.6200 & 0.4900 & 3 & $\begin{array}{l}\text { Fernandes et al. } \\
(2011)\end{array}$ & $\begin{array}{l}\text { traditional Portuguese brick } \\
\text { open fireplace }\end{array}$ & 1 \\
\hline golden wattle & 3.5300 & 3.1300 & 0.3400 & 0.2600 & 3 & $\begin{array}{l}\text { Fernandes et al. } \\
(2011)\end{array}$ & $\begin{array}{l}\text { traditional Portuguese brick } \\
\text { open fireplace }\end{array}$ & 1 \\
\hline eucalypt & 5.1100 & 3.9000 & 0.3600 & 0.3600 & 3 & $\begin{array}{l}\text { Fernandes et al. } \\
(2011)\end{array}$ & $\begin{array}{l}\text { traditional Portuguese brick } \\
\text { open fireplace }\end{array}$ & 1 \\
\hline
\end{tabular}




\begin{tabular}{|c|c|c|c|c|c|c|c|c|}
\hline cork oak & 10.0600 & 5.2400 & 0.6800 & 0.4000 & 3 & $\begin{array}{l}\text { Fernandes et al. } \\
(2011)\end{array}$ & $\begin{array}{l}\text { traditional Portuguese brick } \\
\text { open fireplace }\end{array}$ & 1 \\
\hline olive & 9.1000 & 5.7400 & 0.3900 & 0.1600 & 3 & $\begin{array}{l}\text { Fernandes et al. } \\
(2011)\end{array}$ & $\begin{array}{l}\text { traditional Portuguese brick } \\
\text { open fireplace }\end{array}$ & 1 \\
\hline holm oak & 7.2200 & 4.0300 & 0.3000 & 0.1100 & 3 & $\begin{array}{l}\text { Fernandes et al. } \\
(2011)\end{array}$ & $\begin{array}{l}\text { traditional Portuguese brick } \\
\text { open fireplace }\end{array}$ & 1 \\
\hline Portugese oak & 6.0600 & 3.4000 & 0.3200 & 0.2000 & 3 & $\begin{array}{l}\text { Fernandes et al. } \\
(2011)\end{array}$ & $\begin{array}{l}\text { traditional Portuguese brick } \\
\text { open fireplace }\end{array}$ & 1 \\
\hline $\begin{array}{l}\text { Chinese white } \\
\text { poplar }\end{array}$ & 0.6600 & 0.3200 & 0.8800 & 0.4900 & 3 & $\begin{array}{l}\text { Guofeng et al. } \\
(2012)\end{array}$ & brick stove (rural) & 1 \\
\hline elm & 0.7900 & 0.1500 & 1.2000 & 0.3000 & 3 & $\begin{array}{l}\text { Guofeng et al. } \\
(2012)\end{array}$ & brick stove (rural) & 1 \\
\hline yellow locust & 1.9000 & 1.5000 & 0.2100 & 0.1500 & 3 & $\begin{array}{l}\text { Guofeng et al. } \\
(2012)\end{array}$ & brick stove (rural) & 1 \\
\hline maple & 0.1100 & 0.0100 & 0.0560 & 0.0040 & 3 & $\begin{array}{l}\text { Guofeng et al. } \\
(2012)\end{array}$ & brick stove (rural) & 1 \\
\hline fir & 0.9700 & 0.8800 & 0.9500 & 0.2000 & 3 & Guofeng et al. & brick stove (rural) & 1 \\
\hline larch & 0.1400 & 0.1100 & 0.3500 & 0.3400 & 3 & $\begin{array}{l}\text { Guofeng et al. } \\
(2012)\end{array}$ & brick stove (rural) & 1 \\
\hline water Chinese fir & 0.3600 & 0.1700 & 0.8500 & 0.4500 & 3 & $\begin{array}{l}\text { Guofeng et al. } \\
(2012)\end{array}$ & brick stove (rural) & 1 \\
\hline cypress & 0.8200 & 0.4500 & 0.7100 & 0.3900 & 3 & Guofeng et al. & brick stove (rural) & 1 \\
\hline oak & 0.5400 & 0.6300 & 0.1300 & 0.1300 & 3 & $\begin{array}{l}\text { Guofeng et al. } \\
(2012)\end{array}$ & brick stove (rural) & 1 \\
\hline chinese pine & 0.6000 & 0.3500 & 0.9400 & 0.4000 & 3 & $\begin{array}{l}\text { Guofeng et al. } \\
(2012)\end{array}$ & brick stove (rural) & 1 \\
\hline
\end{tabular}




\begin{tabular}{|c|c|c|c|c|c|c|c|c|c|c|}
\hline willow & 0.2300 & 0.1000 & 0.4700 & 0.3000 & & & 3 & $\begin{array}{l}\text { Guofeng et al. } \\
(2012)\end{array}$ & brick stove (rural) & 1 \\
\hline $\begin{array}{l}\text { Paulownia tomen- } \\
\text { tosa }\end{array}$ & 0.3900 & 0.1200 & 0.9400 & 0.5100 & & & 3 & Guofeng et al. & brick stove (rural) & 1 \\
\hline toon & 0.1900 & 0.1300 & 0.5200 & 0.4100 & & & 3 & $\begin{array}{l}\text { Guofeng et al. } \\
(2012)\end{array}$ & brick stove (rural) & 1 \\
\hline white birch & 0.6900 & 0.3200 & 0.6700 & 0.6100 & & & 3 & $\begin{array}{l}\text { Guofeng et al. } \\
(2012)\end{array}$ & brick stove (rural) & 1 \\
\hline Lespedeza & 0.2100 & 0.1700 & 0.4800 & 0.4000 & & & 3 & Guofeng et al. & brick stove (rural) & 1 \\
\hline Buxus sinica & 1.2000 & 0.1000 & 2.5000 & 1.7000 & & & 3 & $\begin{array}{l}\text { Guofeng et al. } \\
(2012)\end{array}$ & brick stove (rural) & 1 \\
\hline holly & 0.7300 & 0.2800 & 1.6000 & 0.4000 & & & 3 & Guofeng et al. & brick stove (rural) & 1 \\
\hline bamboo & 0.1300 & 0.0900 & 1.2000 & 0.5000 & & & 3 & Guofeng et al. & brick stove (rural) & 1 \\
\hline barley straw & & & & & 0.0400 & 0.0000 & 2 & $\begin{array}{lr}\text { Jenkins } & \text { et al. } \\
(1996) ; & \text { a932- } \\
126 b \_1 & \end{array}$ & wind tunnel & 3 \\
\hline corn stover & & & & & 0.2000 & 0.0141 & 2 & $\begin{array}{l}\text { Jenkins et al. } \\
(1996) ; \\
126 b \_1\end{array}$ & wind tunnel & 3 \\
\hline rice straw & & & & & 0.5910 & 0.2150 & 8 & $\begin{array}{l}\text { Jenkins et al. } \\
(1996) ; \\
126 b \_1\end{array}$ & wind tunnel & 3 \\
\hline wheat straw & & & & & 0.4700 & 0.2758 & 2 & $\begin{array}{l}\text { Jenkins et al. } \\
(1996) ; \\
126 b \_1\end{array}$ & wind tunnel & 3 \\
\hline
\end{tabular}




\begin{tabular}{|c|c|c|c|c|c|c|c|c|c|c|}
\hline $\begin{array}{l}\text { seasoned oak } \\
\text { seasoned pine }\end{array}$ & & & & & $\begin{array}{l}0.1671 \\
0.2531\end{array}$ & 1 & $\begin{array}{l}\text { DeAngel } \\
(1980) \\
\text { DeAngel } \\
(1980\end{array}$ & $\begin{array}{l}\text { s et al. } \\
\text { s et al. }\end{array}$ & $\begin{array}{l}\text { nonbaffled stove } \\
\text { nonbaffled stove }\end{array}$ & 1 \\
\hline $\begin{array}{l}\text { Kentucky Blue- } \\
\text { grass }\end{array}$ & 15.0000 & 5.2000 & 0.5000 & 0.1100 & & 4 & $\begin{array}{l}\text { Holder } \\
(2017)\end{array}$ & et al. & field measurements, Aerostat & 4 \\
\hline $\begin{array}{l}\text { Kentucky Blue- } \\
\text { grass }\end{array}$ & 11.0000 & 6.1000 & 0.3200 & 0.1000 & & 4 & $\begin{array}{l}\text { Holder } \\
(2017)\end{array}$ & et al. & field measurements, ground & 4 \\
\hline $\begin{array}{l}\text { Kentucky Blue- } \\
\text { grass }\end{array}$ & & & 0.7600 & 0.3300 & & 4 & $\begin{array}{l}\text { Holder } \\
(2017)\end{array}$ & et al. & $\begin{array}{l}\text { field measurements, Aerostat, } \\
\text { eBC }\end{array}$ & 4 \\
\hline $\begin{array}{l}\text { Kentucky Blue- } \\
\text { grass }\end{array}$ & & & 0.9300 & 0.0730 & & 4 & $\begin{array}{l}\text { Holder } \\
(2017)\end{array}$ & et al. & $\begin{array}{l}\text { field measurements, ground, } \\
\text { eBC }\end{array}$ & 4 \\
\hline wheat & 9.4000 & 1.6000 & 0.5000 & 0.2000 & & 4 & $\begin{array}{l}\text { Holder } \\
(2017)\end{array}$ & et al. & field measurements, Aerostat & 3 \\
\hline wheat & 11.2000 & 2.5000 & 0.2000 & 0.0000 & & 4 & $\begin{array}{l}\text { Holder } \\
(2017)\end{array}$ & et al. & field measurements, ground & 3 \\
\hline wheat & & & 0.6000 & 0.1000 & & 4 & $\begin{array}{l}\text { Holder } \\
(2017)\end{array}$ & et al. & $\begin{array}{l}\text { field measurements, Aerostat, } \\
\mathrm{eBC}\end{array}$ & 3 \\
\hline wheat & & & 0.5000 & 0.1000 & & 4 & $\begin{array}{l}\text { Holder } \\
(2017)\end{array}$ & et al. & $\begin{array}{l}\text { field measurements, ground, } \\
\text { eBC }\end{array}$ & 3 \\
\hline wheat & & & 0.7000 & 0.0900 & & 8 & Zhang & et al. & $\begin{array}{l}\text { field measurements, EF based } \\
\text { on } \mathrm{CO}_{2}\end{array}$ & 3 \\
\hline rice & & & 0.5600 & 0.0400 & & 4 & $\begin{array}{l}\text { Zhang } \\
(2015)\end{array}$ & et al. & $\begin{array}{l}\text { field measurements, EF based } \\
\text { on } \mathrm{CO}_{2}\end{array}$ & 3 \\
\hline rapeseed & & & 2.8900 & 0.7000 & & 4 & $\begin{array}{l}\text { Zhang } \\
(2015)\end{array}$ & et al. & $\begin{array}{l}\text { field measurements, EF based } \\
\text { on } \mathrm{CO}_{2}\end{array}$ & 3 \\
\hline wheat & & & 0.4300 & 0.1000 & & 8 & $\begin{array}{l}\text { Zhang } \\
(2015)\end{array}$ & et al. & $\begin{array}{l}\text { field measurements, EF based } \\
\text { on } \mathrm{CO}\end{array}$ & 3 \\
\hline
\end{tabular}




\begin{tabular}{|c|c|c|c|c|c|c|c|c|c|c|}
\hline rapeseed & & & $\begin{array}{l}0.2500 \\
1.0100\end{array}$ & $\begin{array}{l}0.1100 \\
0.2700\end{array}$ & & & 4 & $\begin{array}{l}\text { Zhang et al. } \\
(2015) \\
\text { Zhang } \\
(2015)\end{array}$ et al. & $\begin{array}{l}\text { field measurements, EF based } \\
\text { on CO } \\
\text { field measurements, EF based } \\
\text { on CO }\end{array}$ & 3 \\
\hline charcoal burning & 1.7800 & 2.8000 & 0.6500 & 0.3000 & & & 8 & Keita et al. (2018) & $\begin{array}{l}\text { trad. stoves (metal or baked } \\
\text { earth), field meas.; per } \mathrm{kg} \\
\text { charcoal }\end{array}$ & 5 \\
\hline charcoal making & 3.9300 & 1.0100 & 0.2200 & 0.1600 & & & 8 & Keita et al. (2018) & $\begin{array}{l}\text { trad. stoves (metal or baked } \\
\text { earth), field meas.; per kg } \\
\text { wood }\end{array}$ & 6 \\
\hline wood (iroko) & 6.5000 & 1.9800 & 0.5200 & 0.3900 & & & 4 & Keita et al. (2018) & $\begin{array}{l}\text { traditional charcoal-making } \\
\text { furnaces, field meas. }\end{array}$ & 1 \\
\hline wood(hevea) & 15.6100 & 6.4400 & 1.4500 & 0.6100 & & & 4 & Keita et al. (2018) & $\begin{array}{l}\text { traditional charcoal-making } \\
\text { furnaces, field meas. }\end{array}$ & 1 \\
\hline charcoal making & & & & & 0.1860 & 0.1350 & 7 & Park et al. (2013) & $\begin{array}{l}\text { charcoal kiln, field meas.; per } \\
\text { kg wood }\end{array}$ & 6 \\
\hline wheat stubble & 1.9000 & 1.0482 & & & & & 6 & $\begin{array}{l}\text { Dhammapala } \\
\text { et al. (2007a) }\end{array}$ & test burn facility & 3 \\
\hline $\begin{array}{l}\text { Kentucky Blue- } \\
\text { grass stubble }\end{array}$ & 6.9000 & 2.2346 & & & & & 29 & $\begin{array}{l}\text { Dhammapala } \\
\text { et al. (2007a) }\end{array}$ & test burn facility & 4 \\
\hline wheat stubble & & & 0.3500 & 0.2382 & & & 11 & $\begin{array}{l}\text { Dhammapala } \\
\text { et al. (2007a) }\end{array}$ & test burn facility & 3 \\
\hline $\begin{array}{l}\text { Kentucky Blue- } \\
\text { grass stubble }\end{array}$ & & & 0.6300 & 0.1472 & & & 29 & $\begin{array}{l}\text { Dhammapala } \\
\text { et al. (2007a) }\end{array}$ & test burn facility & 4 \\
\hline wheat stubble & 2.7360 & 1.0080 & & & & & 4 & $\begin{array}{l}\text { Dhammapala } \\
\text { et al. }(2007 \mathrm{~b})\end{array}$ & field burning & 3 \\
\hline $\begin{array}{l}\text { Kentucky Blue- } \\
\text { grass stubble }\end{array}$ & 6.9330 & & & & & & 1 & $\begin{array}{l}\text { Dhammapala } \\
\text { et al. }(2007 \mathrm{~b})\end{array}$ & field burning & 4 \\
\hline
\end{tabular}




\begin{tabular}{|c|c|c|c|c|c|c|c|c|c|c|}
\hline $\begin{array}{l}\text { Kentucky Blue- } \\
\text { grass stubble }\end{array}$ & & & $\begin{array}{l}0.3760 \\
0.7980\end{array}$ & $\begin{array}{l}0.2890 \\
0.3440\end{array}$ & & & 2 & $\begin{array}{l}\text { Dhammapala } \\
\text { et al. }(2007 \mathrm{~b}) \\
\text { Dhammapala } \\
\text { et al. }(2007 \mathrm{~b})\end{array}$ & $\begin{array}{l}\text { field burning } \\
\text { field burning }\end{array}$ & 4 \\
\hline grass, ground & 7.0000 & & 0.6200 & & & & 1 & $\begin{array}{l}\text { Strand et al. } \\
(2016)\end{array}$ & ground & 4 \\
\hline grass, aerostat & 6.5000 & & 0.5600 & & & & 1 & $\begin{array}{l}\text { Strand et al. } \\
(2016)\end{array}$ & Aerostat & 4 \\
\hline grass, ground & & & 1.1000 & & & & 1 & $\begin{array}{l}\text { Strand et al. } \\
(2016)\end{array}$ & ground; continuous eBC & 4 \\
\hline grass, aerostat & & & 0.9100 & & & & 1 & $\begin{array}{l}\text { Strand et al. } \\
(2016)\end{array}$ & Aerostat; continuous eBC & 4 \\
\hline grass (Brazil) & 5.1000 & 2.2500 & 0.6500 & 0.4500 & 0.4400 & 0.1800 & 6 & Ferek et al. (1998) & airborne measurements & 4 \\
\hline fuel wood, Delhi & 0.8500 & 0.7000 & 0.3700 & 0.1800 & & & 101 & Saud et al. (2012) & dilution sampler & 1 \\
\hline fuel wood, Punjab & 0.9300 & 0.9600 & 0.4400 & 0.2900 & & & 139 & Saud et al. (2012) & dilution sampler & 1 \\
\hline $\begin{array}{l}\text { fuel wood, } \\
\text { Haryana }\end{array}$ & 0.7800 & 0.4100 & 0.4200 & 0.0700 & & & 92 & Saud et al. (2012) & dilution sampler & 1 \\
\hline $\begin{array}{l}\text { fuel wood, Uttar } \\
\text { Pradesh }\end{array}$ & 1.3200 & 1.0000 & 0.3100 & 0.1200 & & & 149 & Saud et al. (2012) & dilution sampler & 1 \\
\hline $\begin{array}{l}\text { fuel wood, Ut- } \\
\text { tarakhand }\end{array}$ & 0.9200 & 0.5400 & 0.2700 & 0.0800 & & & 181 & Saud et al. (2012) & dilution sampler & 1 \\
\hline fuel wood, Bihar & 1.2800 & 0.9900 & 0.3600 & 0.1900 & & & 85 & Saud et al. (2012) & dilution sampler & 1 \\
\hline $\begin{array}{l}\text { fuel wood, West } \\
\text { Bengal }\end{array}$ & 0.5500 & 0.7000 & 0.2500 & 0.1300 & & & 19 & Saud et al. (2012) & dilution sampler & 1 \\
\hline $\begin{array}{l}\text { crop residue, } \\
\text { Delhi }\end{array}$ & 2.1000 & 1.4400 & 0.5700 & 0.3600 & & & 20 & Saud et al. (2012) & dilution sampler & 2 \\
\hline $\begin{array}{l}\text { crop residue, } \\
\text { Punjab }\end{array}$ & 0.5600 & 0.2800 & 0.2500 & 0.0700 & & & 40 & Saud et al. (2012) & dilution sampler & 2 \\
\hline
\end{tabular}




\begin{tabular}{|c|c|c|c|c|c|c|c|c|}
\hline crop residue, & 0.9400 & 0.7100 & 0.4200 & 0.1300 & 35 & Saud et al. (2012) & dilution sampler & 2 \\
\hline Haryana & & & & & & & & \\
\hline $\begin{array}{l}\text { crop residue, Ut- } \\
\text { tar Pradesh }\end{array}$ & 2.3400 & 1.2800 & 0.3900 & 0.1900 & 107 & Saud et al. (2012) & dilution sampler & 2 \\
\hline $\begin{array}{l}\text { crop residue, } \mathrm{Bi}- \\
\text { har }\end{array}$ & 1.8700 & 1.6600 & 0.4300 & 0.5200 & 105 & Saud et al. (2012) & dilution sampler & 2 \\
\hline crop residue, & 0.9700 & 0.8800 & 0.1800 & 0.1100 & 20 & Saud et al. (2012) & dilution sampler & 2 \\
\hline West Bengal & & & & & & Soud ot ol (2012) & & \\
\hline dung cake, Delhi & 4.5100 & 1.3400 & 0.9000 & 0.3500 & 95 & Saud et al. (2012) & dilution sampler & 2 \\
\hline $\begin{array}{l}\text { dung cake, Pun- } \\
\text { jab }\end{array}$ & 4.6400 & 0.7800 & 0.5900 & 0.2400 & 48 & Saud et al. (2012) & dilution sampler & 2 \\
\hline dung cake, & 3.7800 & 0.4700 & 0.5400 & 0.3400 & 38 & Saud et al. (2012), & dilution sampler & 2 \\
\hline Haryana & & & & & & & & \\
\hline $\begin{array}{l}\text { dung cake, Uttar } \\
\text { Pradesh }\end{array}$ & 4.4100 & 1.1900 & 0.4100 & 0.2100 & 45 & Saud et al. (2012) & dilution sampler & 2 \\
\hline dung cake, Bihar & 4.1400 & 1.4800 & 0.2800 & 0.2100 & 68 & Saud et al. (2012) & dilution sampler & 2 \\
\hline $\begin{array}{l}\text { dung cake, West } \\
\text { Bengal }\end{array}$ & 1.7400 & 1.1000 & 0.2200 & 0.1300 & 8 & Saud et al. (2012) & dilution sampler & 2 \\
\hline rice straw & 2.7838 & 1.1874 & 0.4789 & 0.2891 & 4 & Oanh et al. (2011) & field burning & 3 \\
\hline wheat straw & 2.6000 & 1.8000 & 0.4600 & 0.1900 & 3 & Wei et al. $(2014)$ & $\begin{array}{l}\text { improved two-pot stove with } \\
\text { chimney, 1-year old }\end{array}$ & 2 \\
\hline rape straw & 0.5800 & 0.1700 & 0.4700 & 0.4800 & 3 & Wei et al. (2014) & $\begin{array}{l}\text { improved two-pot stove with } \\
\text { chimney, 1-year old }\end{array}$ & 2 \\
\hline rice straw & 1.1000 & 0.9000 & 0.5100 & 0.3700 & 3 & Wei et al. (2014) & $\begin{array}{l}\text { improved two-pot stove with } \\
\text { chimney, 1-year old }\end{array}$ & 2 \\
\hline cotton straw & 3.1000 & 4.5000 & 1.2000 & 1.4000 & 3 & Wei et al. (2014) & $\begin{array}{l}\text { improved two-pot stove with } \\
\text { chimney, 1-year old }\end{array}$ & 2 \\
\hline wheat straw & 4.1000 & 1.8000 & 1.4000 & 0.9000 & 3 & Wei et al. (2014) & $\begin{array}{l}\text { improved two-pot stove with } \\
\text { chimney, } 15 \text {-year old }\end{array}$ & 2 \\
\hline
\end{tabular}




\begin{tabular}{|c|c|c|c|c|c|c|c|c|c|c|}
\hline rape straw & 4.9000 & 6.5000 & 2.7000 & 2.4000 & & & 3 & Wei et al. (2014) & improved two-pot stove with & 2 \\
\hline rice straw & 1.5000 & 0.6000 & 0.6400 & 0.1900 & & & 3 & Wei et al. (2014) & improved two-pot stove with & 2 \\
\hline cotton straw & 2.5000 & 1.8000 & 0.9600 & 0.9000 & & & 3 & Wei et al. (2014) & $\begin{array}{l}\text { improved two-pot stove with } \\
\text { chimney, } 15 \text {-year old }\end{array}$ & 2 \\
\hline wheat straw & 4.0659 & 0.3297 & 0.3187 & 0.0220 & & & 3 & Tian et al. (2017) & traditional stove & 2 \\
\hline maize straw & 7.0330 & 1.9780 & 0.5275 & 0.0769 & & & 3 & Tian et al. (2017) & traditional stove & 2 \\
\hline rice straw & 8.4615 & 1.4286 & 0.5714 & 0.0659 & & & 3 & Tian et al. (2017) & traditional stove & 2 \\
\hline charcoal burning & & & & & 0.5000 & 0.3000 & 1 & Gadi et al. $(2011)$ & U-shaped chimney & 5 \\
\hline
\end{tabular}




\section{References}

AIRUSE LIFE (2016). Emission factors for biomass burning, Report 9. Tech. rep. AIRUSE LIFE $11 \mathrm{ENV} / \mathrm{ES} / 584$.

Alves, C., C. Gonçalves, A. P. Fernandes, L. Tarelho, and C. Pio (2011). "Fireplace and woodstove fine particle emissions from combustion of western Mediterranean wood types". In: Atmos. Res. 101.3. International Conference on Nucleation and Atmospheric Aerosols (Part 2), pp. 692-700. ISSN: 0169-8095. DOI: https://doi.org/10.1016/j.atmosres.2011.04.015. URL: http://www.sciencedirect.com/science/article/pii/S0169809511001268.

Bond, T. C., D. G. Streets, K. F. Yarber, S. M. Nelson, J.-H. Woo, and Z. Klimont (2004). "A technology-based global inventory of black and organic carbon emissions from combustion". In: J. Geophys. Res.-Atmos. 109.D14. DOI: 10.1029/2003JD003697, eprint: https: //agupubs . onlinelibrary.wiley.com/doi/pdf/10.1029/2003JD003697, URL: https: //agupubs.onlinelibrary.wiley.com/doi/abs/10.1029/2003JD003697.

Calvo, A., V. Martins, T. Nunes, M. Duarte, R. Hillamo, K. Teinilä, V. Pont, A. Castro, R. Fraile, L. Tarelho, and C. Alves (2015). "Residential wood combustion in two domestic devices: Relationship of different parameters throughout the combustion cycle". In: Atmos. Environ. 116, pp. 72-82. ISSN: 1352-2310. DOI: https://doi.org/10.1016/j . atmosenv.2015.06. 012. URL: http://www.sciencedirect.com/science/article/pii/S1352231015301552.

Cao, G., X. Zhang, S. Gong, and F. Zheng (2008). "Investigation on emission factors of particulate matter and gaseous pollutants from crop residue burning". In: J. Environ. Sci. 20, pp. $50-55$.

DeAngelis, D. G., D. S. Ruffin, and R. B. Reznik (1980). Preliminary Characterization of Emissions from Wood-fired Residential Combustion Equipment. U.S. Environmental Protection Agency, EPA-600/7-80-040.

Dhammapala, R., C. Claiborn, J. Jimenez, J. Corkill, B. Gullett, C. Simpson, and M. Paulsen (2007a). "Emission factors of PAHs, methoxyphenols, levoglucosan, elemental carbon and organic carbon from simulated wheat and Kentucky bluegrass stubble burns". In: Atmos. Environ. 41, pp. 2660-2669.

Dhammapala, R., C. Claiborn, C. Simpson, and J. Jimenez (2007b). "Emission factors from wheat and Kentucky bluegrass stubble burning: Comparison of field and simulated burn experiments". In: Atmos. Environ. 41, pp. 1512-1520.

Ferek, R. J., J. S. Reid, P. V. Hobbs, D. R. Blake, and C. Liousse (1998). "Emission factors of hydrocarbons, halocarbons, trace gases and particles from biomass burning in Brazil". In: $J$. Geophys. Res. 103.D24, pp. 32107-32118.

Fernandes, A. P., C. Alves, C. Gonçalves, L. Tarelho, C. Pio, C. Schimdl, and H. Bauer (2011). "Emission factors from residential combustion appliances burning Portuguese biomass fuels". In: J. Environ. Monit. 11, pp. 3196-3206. DOI: 10.1039/c1em10500k.

Gadi, R., U. C. Kulshrestha, A. K. Sarkar, S. C. Garg, and D. C. Parashar (2011). "Emissions of $\mathrm{SO}_{2}$ and $\mathrm{NO}_{x}$ from biofuels in India". In: Tellus B: Chemical and Physical Meteorology 55.5, pp. 787-795. DOI: 10.3402/tellusb.v55i3.16370.

Goodchild, H. (2007). "Modelling Roman agricultural production in the middle Tiber Valley, Central Italy". PhD thesis. Institute of Archaeology and Antiquity, School of Historical Studies, The University of Birmingham.

Guofeng, S., W. Siye, W. Wen, Z. Yanyan, M. Yujia, W. Bin, W. Rong, L. Wei, S. Huizhong, H. Ye, Y. Yifeng, W. Wei, W. Xilong, W. Xuejun, and T. Shu (Apr. 2012). "Emission Factors, Size Distributions, and Emission Inventories of Carbonaceous Particulate Matter from Res- 
idential Wood Combustion in Rural China". In: Environ. Sci. Technol. 46.7, pp. 4207-4214. ISSN: 0013-936X. DOI: 10.1021/es203957u. URL: https://doi.org/10.1021/es203957u.

Habib, G., C. Venkataraman, T. C. Bond, and J. J. Schauer (2008). "Chemical, Microphysical and Optical Properties of Primary Particles from the Combustion of Biomass Fuels". In: Environ. Sci. Technol. 42, pp. 8829-8834.

Hamilton, D., S. Hantson, C. Scott, J. Kaplan, K. Pringle, L. Nieradzik, A. Rap, G. Folberth, D. Spracklen, and K. Carslaw (Dec. 2018). "Reassessment of pre-industrial fire emissions strongly affects anthropogenic aerosol forcing". English. In: Nat. Commun. 9.1. ISSN: 20411723. DOI: $10.1038 / \mathrm{s} 41467-018-05592-9$.

Harris, W. (2013). "Defining and Detecting Mediterranean Deforestation, 800BCE to 700CE". In: The Ancient Mediterranean Environment between Science and History. Leiden, The Netherlands: Brill, pp. 173-194. ISBN: 9789004254053. URL: https ://brill .com/view/ book/edcoll/9789004254053/B9789004254053_008.xml.

Hays, M. D., P. M. Fine, C. D. Geron, M. J. Kleeman, and B. K. Gullett (2005). "Open burning of agricultural biomass: Physical and chemical properties of particle-phase emissions". In: Atmos. Environ. 39, pp. 6747-6764.

Holder, A., B. Gullett, S. Urbanski, R. Elleman, S. O'Neill, D. Tabor, W. Mitchell, and K. Baker (2017). "Emissions from prescribed burning of agricultural fields in the Pacific Northwest". In: Atmos. Environ. 166, pp. 22-33. ISSN: 1352-2310. DOI: https://doi.org/10.1016/j. atmosenv.2017.06.043. URL: http://www.sciencedirect.com/science/article/pii/ S1352231017304247.

Hopkins, K. (2017). "Models, Ships and Staples". In: Sociological Studies in Roman History. Ed. by C. Kelly. Cambridge Classical Studies. Cambridge University Press, pp. 213-268. DOI: 10.1017/CB09781139093552.009.

Jenkins, B. M., S. Q. Turn, R. B. Williams, M. Goronea, H. Abd-el-Fattah, J. Mehlschau, N. Raubach, D. P. Y. Chang, M. Kang, S. V. Teague, O. G. Raabe, D. E. Campbell, T. A. Cahill, L. Pritchett, J. Chow, and A. D. Jones (1996). Atmospheric Pollutant Emission Factors From Open Burning of Agricultural and Forest Biomass by Wind Tunnel Simulations: Volume 1-3. Research rep. California Environmental Protection Agency, contract NO. A932-126.

Jimenez, J. R., C. S. Claiborn, R. S. Dhammapala, and C. D. Simpson (2007). "Methoxyphenols and Levoglucosan Ratios in PM2.5 from Wheat and Kentucky Bluegrass Stubble Burning in Eastern Washington and Northern Idaho". In: Environ. Sci. Technol. 41, pp. 7824-7829.

Keita, S., C. Liousse, V. Yoboué, P. Dominutti, B. Guinot, E.-M. Assamoi, A. Borbon, S. L. Haslett, L. Bouvier, A. Colomb, H. Coe, A. Akpo, J. Adon, J. Bahino, M. Doumbia, J. Djossou, C. Galy-Lacaux, E. Gardrat, S. Gnamien, J. F. Léon, M. Ossohou, E. T. N'Datchoh, and L. Roblou (2018). "Particle and VOC emission factor measurements for anthropogenic sources in West Africa". In: Atmos. Chem. Phys. 18.10, pp. 7691-7708. Dor: 10.5194/acp18-7691-2018. URL: https://www .atmos-chem-phys.net/18/7691/2018/.

Li, C., Y. Hu, F. Zhang, J. Chen, Z. Ma, X. Ye, X. Yang, L. Wang, X. Tang, R. Zhang, M. Mu, G. Wang, H. Kan, X. Wang, and A. Mellouki. (2017). "Multi-pollutant emissions from the burning of major agricultural residues in China and the related health-economic effects". In: Atmos. Chem. Phys. 17, pp. 4957-4988.

Lohri, C. R., H. M. Rajabu, D. J. Sweeney, and C. Zurbrügg (2016). "Char fuel production in developing countries - A review of urban biowaste carbonization". In: Renewable Sustainable Energy Rev. 59, pp. 1514-1530. ISSN: 1364-0321. DOI: https:// doi .org/10.1016/j . rser .2016.01.088. URL: http://www . sciencedirect.com/science/article/pii/ S1364032116001180. 
McDonald, J. D., B. Zielinska, E. M. Fujita, J. C. Sagebiel, J. C. Chow, and J. G. Watson (2000). "Fine Particle and Gaseous Emission Rates from Residential Wood Combustion". In: Environ. Sci. Technol. 34.11, pp. 2080-2091. DOI: 10.1021/es9909632, eprint: https: //doi.org/10.1021/es9909632. URL: https://doi.org/10.1021/es9909632.

Mietz, M. (2016). "The fuel economy of public bathhouses in the Roman Empire". MA thesis. Faculty of Arts and Philosophy, Campus Boekentoren, Blandijnberg 2, B-9000 Ghent, Belgium: Ghent University.

Oanh, N. T. K., B. T. Ly, D. Tipayarom, B. R. Manandhar, P. Prapat, C. D. Simpson, and L.-J. S. Liu (2011). "Characterization of particulate matter emission from open burning of rice straw". In: Atmos. Environ. 45, pp. 493-502.

Olson, S. D. (1991). "Firewood and charcoal in Classical Athens". In: Hesperia: The Journal of the American of Classical Studies at Athens 60.3, pp. 411-420.

Pandey, A., S. Patel, S. Pervez, S. Tiwari, G. Yadama, J. C. Chow, J. G. Watson, P. Biswas, and R. K. Chakrabarty (2017). "Aerosol emissions factors from traditional biomass cookstoves in India: insights from field measurements". In: Atmos. Chem. Phys. 17, pp. 13721-13729.

Park, S.-K., S.-J. Choi, J.-Y. Kim, G.-J. Park, U.-H. Hwang, J.-J. Lee, and T.-S. Kim (2013). “A Field Survey on the Characteristics of Air Pollutants Emission from Commercial Charcoal Kiln". In: J. Kosae 29.5, pp. 601-614.

Rowan, E. (2015). "Olive oil pressing waste as a fuel source in Antiquity". In: Am. J. Archaeol. 119.4, pp. 465-482. DOI: $10.3764 / a j a .119 .4 .0465$.

Sahai, S., C. Sharma, D. P. Singh, C. K. Dixit, N. Singh, P. Sharma, K. Singh, S. Bhatt, S. Ghude, V. Gupta, R. K. Gupta, M. K. Tiwari, S. C. Garg, A. P. Mitra, and P. K. Gupta (2007). "A study for development of emission factors for trace gases and carbonaceous particulate species from in situ burning of wheat straw in agricultural fields in india”. In: Atmos. Environ. 41, pp. 9173-9186.

Saud, T., R. Gautam, T. K. Mandal, S. Gadi R., S. D. P., S. K., M. Dahiya, and M. Saxena (2012). "Emission estimates of organic and elemental carbon from household biomass fuel used over the Indo-Gangetic Plain (IGP), India". In: Atmos. Environ. 61, pp. 212-220.

Shen, G., Y. Yang, W. Wang, S. Tao, C. Zhu, Y. Min, M. Xue, J. Ding, B. Wang, R. Wang, H. Shen, W. Li, X. Wang, and A. G. Russell (Sept. 2010). "Emission Factors of Particulate Matter and Elemental Carbon for Crop Residues and Coals Burned in Typical Household Stoves in China". In: Environ. Sci. Technol. 44.18, pp. 7157-7162. ISSN: 0013-936X. DOI: 10.1021/es101313y, URL: https://doi.org/10.1021/es101313y.

Sinclair, T. R. (1998). "Historical Changes in Harvest Index and Crop Nitrogen Accumulation. Review and Interpretation". In: Crop Sci. 38.3.

Smith, K. R. (2000). Greenhouse gases from small-scale combustion devices in developing countries Phase IIa. Household Stoves in India. Tech. rep. U.S. Environmental Protection Agency.

Spurr, M. S. (1986). Arable cultivation in Roman Italy. c.200 B.C.-c.A.D. 100. 31-34 Gordon Square, London WC1H 0PP: The Society for the Promotion of Roman Studies.

Strand, T., B. Gullett, S. Urbanski, S. O’Neill, B. Potter, J. Aurell, A. Holder, N. Larkin, M. Moore, and M. Rorig (2016). "Grassland and forest understorey biomass emissions from prescribed fires in the southeastern United States - RxCADRE 2012". In: Int. J. Wildland Fire 25, pp. 102-113.

Tataryn (2016). The Roman Empire and its clients in 117 AD during the reign of emperor Trajan. Ed. by Wikimedia Commons, retrieved 14 Nov 2018. URL: https://commons.wikimedia. org/wiki/File:Roman_Empire_Trajan_117AD.png. 
Temin, P. (2006). "The Economy of the Early Roman Empire". In: J. Econ. Perspect. 20.1, pp. 133-151.

Tian, J., H. Ni, J. Cao, Y. Han, Q. Wang, X. Wang, L.-W. Chen, J. C. Chow, J. G. Watson, C. Wei, J. Sun, T. Zhang, and R. Huang (2017). "Characteristics of carbonaceous particles from residential coal combustion and agricultural biomass burning in China". In: Atmos. Pollut. Res. 8.3, pp. 521-527. ISSN: 1309-1042. DOI: https://doi.org/10.1016/j .apr.2016.12. 006 . URL: http://www.sciencedirect.com/science/article/pii/S1309104216302896.

Turn, S. Q., B. M. Jenkins, J. C. Chow, L. C. Pritchett, D. Campbell, T. Cahill, and S. A. Whalen (1997). "Elemental characterizationof particulate matter emitted from biomass burning: Wind tunnel derived source profiles for herbaceous and wood fuels". In: J. Geophys. Res. 102.D3, pp. 3683-3699.

Van Marle, M. J. E., S. Kloster, B. I. Magi, J. R. Marlon, A.-L. Daniau, R. D. Field, A. Arneth, M. Forrest, S. Hantson, N. M. Kehrwald, W. Knorr, G. Lasslop, F. Li, S. Mangeon, C. Yue, J. W. Kaiser, and G. R. van der Werf (2017). "Historic global biomass burning emissions for CMIP6 (BB4CMIP) based on merging satellite observations with proxies and fire models (1750-2015)". In: Geosci. Model Dev. 10.9, pp. 3329-3357. Dor: 10.5194/gmd-10-33292017. URL: https://www.geosci-model-dev.net/10/3329/2017/

Veal, R. (2017). "Wood and Charcoal for Rome: Towards an Understanding of Ancient Regional Fuel Economics". In: The Economic Integration of Roman Italy. Leiden, The Netherlands: Brill, pp. 388-406. ISBN: 9789004345027. URL: https://brill.com/view/book/edcoll/ 9789004345027/B9789004345027_017.xml.

Wei, S., G. Shen, Y. Zhang, M. Xue, H. Xie, P. Lin, Y. Chen, X. Wang, and S. Tao (2014). "Field measurement on the emissions of PM, OC, EC and PAHs from indoor crop straw burning in rural China”. In: Environ. Pollut. 184, pp. 18-24. ISSN: 0269-7491. DOI: https: //doi.org/10.1016/j.envpol.2013.07.036. URL: http://www .sciencedirect.com/ science/article/pii/S0269749113004120.

Wood, T. S. and S. Baldwin (1985). "Fuelwood and charcoal use in developing countries". In: Ann. Rev. Energy 10, pp. 407-429.

Yevich, R. and J. A. Logan (2003). "An assessment of biofuel use and burning of agricultural waste in the developing world". In: Global Biogeochem. Cycles 17.4. Dor: 10.1029/ 2002GB001952.

Zhang, J., K. Smith, Y. Ma, S. Ye, F. Jiang, W. Qi, P. Liu, M. Khalil, R. Rasmussen, and S. Thorneloe (2000). "Greenhouse gases and other airborne pollutants from household stoves in China: a database for emission factors". In: Atmos. Environ. 34.26, pp. 4537-4549. ISSN: 1352-2310. DOI: https : / / doi . org/10 .1016/S1352-2310(99) 00450-1. URL: http : //www.sciencedirect.com/science/article/pii/S1352231099004501.

Zhang, T., M. J. Wooster, D. C. Green, and B. Main (2015). "New field-based agricultural biomass burning trace gas, PM2.5, and black carbon emission ratios and factors measured in situ at crop residue fires in Eastern China". In: Atmos. Environ. 121. Interdisciplinary Research Aspects of Open Biomass Burning and its Impact on the Atmosphere, pp. 2234. ISSN: 1352-2310. DOI: https://doi.org/10.1016/j . atmosenv. 2015.05.010. URL: http://www.sciencedirect.com/science/article/pii/S1352231015300807. 